\title{
Multi-triplet bound states and finite-temperature dynamics in highly frustrated quantum spin ladders
}

\author{
Andreas Honecker, ${ }^{1}$ Frédéric Mila, ${ }^{2}$ and B. Normand ${ }^{3}$ \\ ${ }^{1}$ Laboratoire de Physique Théorique et Modélisation, CNRS UMR 8089, Université de Cergy-Pontoise, \\ F-95302 Cergy-Pontoise Cedex, France \\ ${ }^{2}$ Institute of Physics, Ecole Polytechnique Fédérale Lausanne (EPFL), CH-1015 Lausanne, Switzerland \\ ${ }^{3}$ Department of Physics, Renmin University of China, Beijing 100872, People's Republic of China \\ (Received 2 June 2016; revised manuscript received 29 July 2016; published 2 September 2016)
}

\begin{abstract}
Low-dimensional quantum magnets at finite temperatures present a complex interplay of quantum and thermal fluctuation effects in a restricted phase space. While some information about dynamical response functions is available from theoretical studies of the one-triplet dispersion in unfrustrated chains and ladders, little is known about the finite-temperature dynamics of frustrated systems. Experimentally, inelastic neutron scattering studies of the highly frustrated two-dimensional material $\mathrm{SrCu}_{2}\left(\mathrm{BO}_{3}\right)_{2}$ show an almost complete destruction of the one-triplet excitation band at a temperature only $1 / 3$ of its gap energy, accompanied by strong scattering intensities for apparent multi-triplet excitations. We investigate these questions in the frustrated spin ladder and present numerical results from exact diagonalization for the dynamical structure factor as a function of temperature. We find anomalously rapid transfer of spectral weight out of the one-triplet band and into both broad and sharp spectral features at a wide range of energies, including below the zero-temperature gap of this excitation. These features are multi-triplet bound states, which develop particularly strongly near the quantum phase transition, fall to particularly low energies there, and persist all the way to infinite temperature. Our results offer valuable insight into the physics of finite-temperature spectral functions in $\mathrm{SrCu}_{2}\left(\mathrm{BO}_{3}\right)_{2}$ and many other highly frustrated spin systems.
\end{abstract}

DOI: 10.1103/PhysRevB.94.094402

\section{INTRODUCTION}

Quantum mechanics mandates a degree of uncertainty in the properties of a physical system. In many-body systems, this uncertainty is manifest as quantum fluctuations between different, and often classically inspired, states of the system or its subcomponents. One-dimensional (1D) quantum antiferromagnets provide an excellent example of a situation where the classical state, which would be Néel order, is destroyed completely by quantum fluctuations, replaced by gapped or gapless states with complex correlations (or "entanglement") but no magnetic order [1]. The canonical gapless 1D quantum magnet, the $S=1 / 2$ Heisenberg chain, has quasi-long-ranged spin correlations and massless, fractionalized "spinon" excitations [2]; its gapped counterparts, including the two-leg $S=1 / 2$ spin ladder [3-5], the $S=1$ "Haldane" chain [6], and the frustrated $S=1 / 2\left(J_{1}-J_{2}\right.$, "Majumdar-Ghosh") chain [7-9], all show exponentially decaying spin correlations, accompanied in the first two cases by robust triplet excitations and in the third by massive spinons. These properties are consequences purely of quantum spin fluctuations in the restricted phase space available in one spatial dimension, and significant progress has been made over the last two decades both in their theoretical description and in their experimental observation [10].

Far less well understood is the additional effect of finite temperatures on these systems. The problem of describing the combination of quantum and thermal fluctuations within the same restricted phase space, to deduce the consequences for the ground and excited states, has proven difficult to address. In the most straightforward description of the triplet excitations of a two-leg spin ladder as robust $\delta$-function peaks in energy [11], a significant band-narrowing effect is obtained as the temperature is increased, and this was in fact observed in the 3D coupled dimer system $\mathrm{TlCuCl}_{3}$ [12]. In lower dimensions, however, more sophisticated means of modeling thermal effects in unfrustrated spin chains suggest rather a systematic and asymmetric broadening of peak widths [13-17], driven by a mixing of available states within the triplet band, and this is confirmed by recent observations in the alternating spin-chain materials $\mathrm{Cu}\left(\mathrm{NO}_{3}\right)_{2} \cdot 2.5 \mathrm{D}_{2} \mathrm{O}$ [18] and $\mathrm{BaCu}_{2} \mathrm{~V}_{2} \mathrm{O}_{8}$ [19]. By contrast, the situation in frustrated 1D systems at finite temperatures remains essentially unexplored.

While systematic experimental data for frustrated spin chains are not yet available, the $2 \mathrm{D}$ material $\mathrm{SrCu}_{2}\left(\mathrm{BO}_{3}\right)_{2}$ offers some crucial insight into the effects of competing interactions. The $S=1 / 2 \mathrm{Cu}^{2+}$ ions in $\mathrm{SrCu}_{2}\left(\mathrm{BO}_{3}\right)_{2}$ form the "Shastry-Sutherland" geometry [20] of "rung" bonds bridging the opposite corners of a square lattice, and at large values of the rung coupling there is an exact ground state of dimer singlets on these bonds. A triplet excitation of any single dimer experiences complete frustration (up to sixth order in a perturbative expansion [21,22]) and therefore the excitation band measured at low temperatures is almost completely flat, at an energy of approximately $3.0 \mathrm{meV}(\simeq 35 \mathrm{~K})$ [23]. Remarkably, if the temperature is increased to only $6 \mathrm{~K}$, this mode suffers a serious loss of intensity, and by $12 \mathrm{~K}$ it is essentially indiscernible, its spectral weight spread over the entire energy spectrum $[24,25]$. Some phenomenological proposals $[24,25]$ and one numerical study [26] have tried to account for this anomalously strong decay of the one-triplet excitation, but with limited success.

We begin with the hypothesis that this behavior is the consequence of bound-state formation, which is strongly enhanced by the presence of frustration. We comment that the Hamiltonian for $\mathrm{SrCu}_{2}\left(\mathrm{BO}_{3}\right)_{2}$ does contain nontrivial 
additional terms, specifically Dzyaloshinskii-Moriya interactions, whose effects would need to be computed in a quantitative treatment of triplet decay. However, our aim is to test the concept that the formation of (many) strongly bound states and the resulting strong enhancement of one-triplet decay may be generic to the finite-temperature dynamical response of frustrated quantum magnets. As a candidate system for the demonstration of a nontrivial thermal redistribution of spectral weight, we investigate the fully frustrated $S=1 / 2$ two-chain spin ladder, introduced in Sec. II; this model is well suited for our study because of the existence of exact multi-triplet bound states over the full range of parameters giving a rung-singlet ground state.

Computing the dynamical spectral function of a lowdimensional quantum spin system at finite temperatures is a challenging problem, requiring knowledge both of the full excitation spectrum and of all matrix elements. Although some analytical progress has been possible by Bethe-Ansatz techniques [14] and by a recent diagrammatic approach $[16,17]$, the applicability of these methods to frustrated systems is limited. Among the available numerical techniques, exact diagonalization (ED) has in the past $[13,18,26]$ been the only fully systematic approach for all models, parameters, and temperatures. Quantum Monte Carlo (QMC) methods are able to deliver dynamical response functions for the lowest excitations of unfrustrated systems, but are in general unsuitable for frustrated ones because of the sign problem. Density-matrixrenormalization-group (DMRG) techniques have previously been of limited value in dealing with the combination of time (dynamics) and temperature, but we comment that recent advances in methodology have the potential to expand very significantly the study of finite-temperature response functions and we review these briefly in Sec. III for their pedagogical value. For the present study, the intrinsic advantages of ED far outweigh its traditional limitation to small system sizes, as we discuss in detail in Sec. III.

Significant information in support of bound-state effects in frustrated systems can be obtained from their thermodynamic response. The calculation of quantities such as the specific heat and susceptibility of a quantum system is also a hard problem, requiring again the full excitation spectrum. Once again, analytical assistance is limited to the spin- $1 / 2$ Heisenberg chain [27-32], also one of the first systems to which ED was applied [33], and beyond this model a variety of numerical methods have been employed for different $1 D$ systems. In parallel with the present study, we have performed a systematic analysis of the magnetic specific heat and susceptibility of the frustrated spin ladder in Ref. [34], to which we refer the reader for full details. Here we draw attention only to the use of ED methods to obtain the thermodynamic response of frustrated spin chains, specifically $J_{1}-J_{2}$ models motivated by certain $S=1 / 2$ and 1 materials [35,36]. QMC techniques have been exploited widely for unfrustrated ladders $[37,38]$, but for frustrated systems are stymied by the sign problem except in special cases [34,39,40]. Quantum transfer matrix (QTM)-DMRG is particularly well suited to the extraction of thermodynamic information for 1D systems and has been applied both to unfrustrated $[41,42]$ and to frustrated $\left(J_{1}-J_{2}\right)$ spin chains, where two characteristic energy scales are found [43-46]. However, these studies have largely been restricted to methods and to specific parameter choices, and we are unaware of any systematic investigation of frustration effects. In Ref. [34] we provide such a study for the fully frustrated ladder, which demonstrates clearly the role of bound states in determining the characteristic evolution of the thermodynamic response and the dominant effect of extended multi-triplet bound states close to a quantum critical point; a summary of these results is presented in Sec. II.

The structure and primary results of this article are as follows. In Sec. II, we present the model of the fully frustrated two-leg spin ladder, summarize its phase diagram and the nature of its multi-triplet bound states, and review analytical and numerical results obtained for the thermodynamics of the system. In Sec. III, we show the results of ED calculations of the dynamical spectral function at all temperatures, for a selection of ladders with different coupling ratios and degrees of frustration. Figures 3-8 provide a complete overview of the momentum dependence of the dynamical structure factor and Fig. 5, in particular, demonstrates a very rapid redistribution of spectral weight with increasing temperature. In Sec. IV, we analyze our results by considering the bound-state spectra, scattering matrix elements, and transfer of spectral weight from the one-triplet sector to the multi-triplet bound states that emerge particularly strongly close to the quantum phase transition. Figures 9 to 13 quantify the temperature dependence of the dynamical structure factor and in Fig. 17 we demonstrate that remarkably sharp spectral features survive up to infinite temperatures. Figure 14 shows the thermal evolution of the leading peaks in the spectral function for one value of the exchange ratio, Fig. 15 analyzes the temperature dependence of the one-triplon line for several values of the exchange ratio, demonstrating accelerated thermal suppression close to the quantum phase transition. Figure 16 interprets these findings in terms of emergent, effective temperature scales of the system. Section $\mathrm{V}$ presents a brief summary, while three appendices contain complementary details concerning the analytical treatment of few-triplon bound states (Appendixes A and B) and finite-size effects in the one-triplon spectral weight (Appendix C).

\section{FULLY FRUSTRATED LADDER}

The Hamiltonian of the frustrated $S=1 / 2$ Heisenberg spin ladder represented in Fig. 1 is

$H=\sum_{i} J_{\perp} \vec{S}_{i}^{1} \cdot \vec{S}_{i}^{2}+\sum_{i, m=1,2}\left(J_{\|} \vec{S}_{i}^{m} \cdot \vec{S}_{i+1}^{m}+J_{\times} \vec{S}_{i}^{m} \cdot \vec{S}_{i+1}^{\bar{m}}\right)$,

where $i$ the rung index, $m=1$ and 2 denote the two chains of the ladder, and $\bar{m}$ is the chain opposite to $m$. The spectrum of the ladder is gapped for all finite values of $J_{\perp}$, and for all values $J_{\perp}>2\left\{J_{\|}, J_{\times}\right\}$the spin correlations are dominated by singlets occupying all of the rung bonds. In this regime, a triplet excitation on one of the rungs (to which, for reasons of clarity, we refer henceforth as a "triplon"), as represented in Fig. 1, is a propagating quasiparticle with hard-core-boson nature. However, as the inter-rung frustration, $J_{\times}$, approaches $J_{\|}$, the nature of the ground state can change (below) and at the fully frustrated point, $J_{\times}=J_{\|}$, the triplon band is completely flat, with energy $\omega_{k}=J_{\perp}$ for all wave vectors $k_{\|}$. 


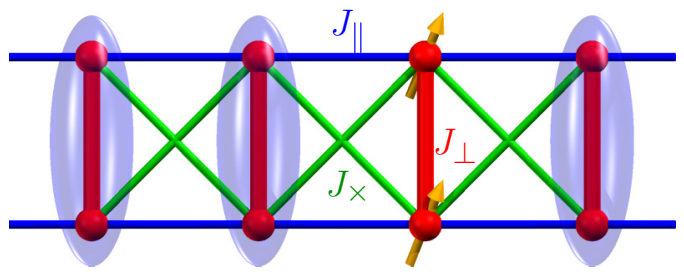

FIG. 1. Representation of superexchange interactions in a frustrated spin ladder (reproduced from Ref. [34]). Each ladder site (spheres) hosts an $S=1 / 2$ quantum spin and the Heisenberg couplings between spins are specified by the parameters $J_{\perp}$ for the ladder rungs, $J_{\|}$for the ladder legs, and $J_{\times}$for the cross-plaquette couplings, which we take to be symmetrical. Purple ellipses represent singlet spin states on the rungs and their absence a rung triplet (center).

The fully frustrated $S=1 / 2$ ladder has been considered in a number of studies. It was first shown in Ref. [47] that, for a range of weak inter-rung couplings, all (one-, two-, threetriplon, ... ) excited states are strictly localized objects, and the critical coupling for this range was found to be $j^{\prime}=J_{\perp} / J_{\|}=$ $J_{\perp} / J_{\times} \geqslant j_{c}^{\prime} \simeq 1.401$. In Ref. [48], it was shown that, up to an additive constant, the Hamiltonian (1) may be expressed as

$$
H / J_{\|}=\sum_{i}\left[j^{\prime}\left(\vec{S}_{i}^{1} \cdot \vec{S}_{i}^{2}-1 / 4\right)+\vec{P}_{i} \cdot \vec{P}_{i+1}\right]
$$

where the first term is finite only for singlet rung states and the second is a Heisenberg interaction between $S=1$ spin operators, $\vec{P}_{i}$, describing the triplon states. A similar expression was obtained in Ref. [49] for the general case of the fully frustrated $m$-leg ladder, where the total spin of each rung remains a good quantum number and thus gives an infinite number of conserved quantities in the thermodynamic limit.

Focusing on the two-leg case, when the inter-rung coupling ratio, $1 / j^{\prime}$, becomes a significant fraction of 1 , it is more favorable for the fully frustrated ladder to abandon the rungsinglet state in favor of a rung-triplet one, thereby satisfying all the inter-rung bonds. The phase diagram of the model has been found [48-50] to have only this one, first-order transition. Because the rung-triplet state has many properties in common with the Haldane chain, one may use Eq. (2) together with the very accurately known ground-state energy of the Haldane chain [51,52] to deduce [48] that $j_{c}^{\prime}=1.401484038971(4)$; the nature of the excited states of the system for $j^{\prime}<j_{c}^{\prime}$ may also be understood in detail from this parallel $[34,48]$. The phase diagram of the frustrated ladder has been discussed in detail in Refs. [47-50,53-56] and has also been considered for related models including tetrahedral cluster chains [57-63].

Returning to the nature of the fully localized excited states, it was shown for the two-leg ladder [47] that these are exact bound states. In the rung-singlet regime at $j^{\prime}>j_{c}^{\prime}$, the properties of an $n$-triplon ladder segment may be deduced by considering the $n$-site Haldane chain with open boundary conditions $[64,65]$. As an example, the excitation with two triplons on neighboring rungs forms an exact bound state consisting of a singlet with energy $E_{2 s} / J_{\|}=2\left(j^{\prime}-1\right)$, a triplet with energy $E_{2 t} / J_{\|}=2 j^{\prime}-1$, and a quintet with $E_{2 q} / J_{\|}=2 j^{\prime}+1$. The spectrum of the analogous 27-branch multiplet for the case $n=3$, which along with $n=2$ will

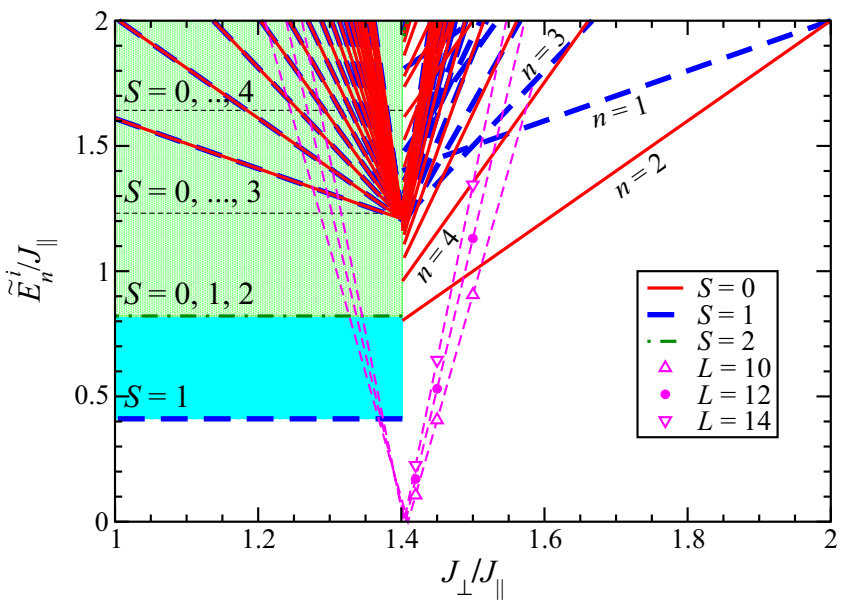

FIG. 2. Schematic representation of the low-lying energy levels $\tilde{E}_{n}^{i}$ of the infinite, fully frustrated spin- $1 / 2$ ladder, shown as a function of $J_{\perp} / J_{\|}$for a broad region around the quantum phase transition at $J_{\perp, c}=1.410484 J_{\|}$. Excitations are classified according to their total spin quantum number, $S$. The additional label $n$ for the rung-singlet phase specifies the origin of the excitations in the bound states of $n$ rung triplons. Shaded regions in the rung-triplet phase denote the onemagnon band and multiple-magnon continua. We indicate in addition the lowest excited energy state appearing close to the transition in our calculations for ladders of $L=10,12$, and 14 rungs; these "intruder states," marked as thin dashed lines, and with their positions at $j^{\prime}=1.42,1.45$, and 1.5 marked by symbols, are finite-size features whose effects are discussed in the text and in Appendix C.

turn out to be most important for our analysis, is presented in Appendix A.

The existence of the exact bound-state regime is a strong asset for the purpose of assessing whether bound or scattering states arising due to frustration effects may contribute to a complex evolution of the dynamical spectral function at finite temperatures, of the type observed in $2 \mathrm{D}$ in $\mathrm{SrCu}_{2}\left(\mathrm{BO}_{3}\right)_{2}$. It is clear by inspection of the energy $E_{2 s}$, the singlet level of the two-triplon bound state, that the gap to the lowest excitation of the system changes from $J_{\perp}$ to $E_{2 s}$ in the parameter region $j_{c}^{\prime}<j^{\prime}<2$. Similarly, the lowest branch of the three-triplon state is a net triplet whose energy, $E_{3 t c}$, becomes the lowest triplet excitation when $j^{\prime}$ falls below 1.5. Thus one may expect immediate and potentially dramatic effects in both the thermodynamic and dynamical response functions of the system in the vicinity of the quantum phase transition.

The energies of the lowest singlet and triplet levels of the exact $n$-triplon bound states at $j^{\prime}=j_{c}^{\prime}$ are shown in Ref. [34] up to $n=20$. Their key property is illustrated in Fig. 2, which we adapt from Fig. 4 of Ref. [34]. As $j^{\prime}$ approaches $j_{c}^{\prime}$, a very large number of excitation branches falls to low (but finite) energy values below the one-triplon gap. The low-lying levels at $j^{\prime}>j_{c}^{\prime}$ are mostly the lowest branches of the $n$-triplon multiplets for all $n$ (Fig. 2), which are respectively singlets or triplets for even or odd $n$ [34]. We will show in Secs. III and IV how these states are responsible for anomalous redistribution of spectral weight at finite temperatures. For $j^{\prime}<j_{c}^{\prime}$, the discrete lines are bound states of singlet excitations within the rung-triplet background, while the shaded areas of Fig. 2 represent the continuous spectrum arising from the magnon 
excitations of the Haldane chain, with different sectors for different magnon numbers.

We stress that Fig. 2 represents the spectrum of the system in the thermodynamic limit, except for the low-lying levels very close to the phase transition. We include as thin, dashed lines the levels on both sides of the transition that lie lower than any others in our finite-size calculations. As discussed in Sec. III, these are performed by ED of the Hamiltonians (1) and (2) for ladders with a finite number, $L$, of rungs and periodic boundary conditions. The levels appearing in Fig. 2 for ladders of $L=10,12$, and 14 rungs are in fact the ground states of the system on the opposite side of the transition and their energies are infinite for systems of infinite length. However, in finite-system calculations, they remain present as spurious "intruder" states, which are low-lying when $\left|j^{\prime}-j_{c}^{\prime}\right| \lesssim 0.05$ (for $L=14$ ). These intruder states are the primary source of finite-size effects in our calculations and their consequences for the physical quantities we compute are discussed in detail in Sec. IV and Appendix C.

Regarding thermodynamic quantities, in Ref. [34], we computed the magnetic specific heat $C(T)$ and susceptibility $\chi(T)$ over the entire range of fully frustrated coupling ratios in Eqs. (1) and (2), spanning the rung-singlet and -triplet regimes. ED methods are appropriate for all values of $j^{\prime}$ away from the phase transition, where the correlation lengths are far shorter than the accessible system size ( $L=14$ rungs). Close to $j^{\prime}=j_{c}^{\prime}$, we used a quantum Monte Carlo technique, which we adapted to be free of the sign problem affecting most frustrated systems, to access sizes up to $L=200$ and thereby obtain numerically exact results. Qualitatively, the effect of frustration is to redistribute the thermodynamic response to both lower and higher energies, despite the onetriplon band remaining entirely flat, and near the transition a sharp, low-energy peak develops in $C(T)$. In a straightforward description based on 2-, 3-, ..,n-triplon bound states within the rung-singlet chain, multi-triplon effects are observable over a wide range of $j^{\prime}$ values, but become critically important in the regime $j^{\prime} \rightarrow j_{c}^{\prime}$, where many high- $n$ states approach the gap energy (Fig. 2) and the peak of $C(T)$ [the upturn in $\chi(T)$ ] is pushed to very low temperatures. We now begin to investigate the consequences of these bound-state effects for the finite-temperature dynamics of frustrated spin ladders, presenting our methods and results in Sec. III and a detailed analysis in Sec. IV.

\section{FINITE-TEMPERATURE DYNAMICAL SPECTRAL FUNCTIONS}

\section{A. Calculating spectral functions at finite temperatures}

We calculate the dynamical structure factor

$$
S^{z z}(\vec{k}, \omega)=\frac{1}{\pi Z} \sum_{i, j} \operatorname{Im} \frac{e^{-E_{i} / T}\left|\left\langle j\left|S^{z}(\vec{k})\right| i\right\rangle\right|^{2}}{\omega-\left(E_{j}-E_{i}+i \eta\right)},
$$

where $Z$ is the partition function and we set $\hbar$ and $k_{\mathrm{B}}$ to 1 . The Fourier-transformed spin operators are defined by

$$
\vec{S}\left(k_{\|}, k_{\perp}\right)=\frac{1}{\sqrt{2 L}} \sum_{\ell=1}^{L} \sum_{m=1}^{2} \exp \left[i\left(k_{\|} \ell+k_{\perp} m\right)\right] \vec{S}_{\ell}^{m},
$$

where the "transverse momentum," $k_{\perp}$, takes the values 0 (symmetric channel) and $\pi$ (antisymmetric channel). The quantity $\eta$ is a small imaginary part, which appears as a line-broadening in the spectral function; in the limit $\eta \rightarrow 0$, one recovers a representation in terms of $\delta$-functions. As discussed in Appendix B, the full rotational symmetry of the problem in spin space means that it is sufficient to compute $S^{z z}(\vec{k}, \omega)$.

For the calculation of Eq. (3), we employ ED in order to perform a complete investigation of two-chain spin ladder over a broad range of temperature. The high symmetries and exact conservation laws in the fully frustrated case extend the accessible system lengths to $L=16$ ladder rungs and the extremely short spin-spin correlation length of the model makes this an eminently viable size. ED has the further significant advantages [66] of providing spectral functions with arbitrarily fine energy resolution and of working with periodic boundary conditions in Eqs. (1) and (2). Our calculations exploit the conservation of $S^{z}$ and the full translational symmetry, as well as certain aspects of the reflection and spin-inversion symmetries of the ladder.

As noted in Sec. I, recent advances in DMRG techniques [67-69] raise the possibility of qualitative improvements in the computation of finite-temperature spectral functions. Dynamical correlation functions were originally obtained at finite temperatures by applying DMRG to QTMs [70], including the computation of their full real-time evolution [71], and later by the use of minimally entangled typical thermal states (METTS) [72,73]. While most modern DMRG approaches retain the basic structure of purifying the mixed density operator and applying real-time evolution [74-81], and have been used with some success for real materials [82], a further recent development $[66,83]$ is to work directly in frequency space at finite temperature. However, the high degeneracies and many conservation laws of the fully frustrated ladder provide an additional challenge to METTS-based DMRG techniques in accessing the full space of available states. For the present purposes, ED therefore provides the optimal approach for computing the spectrum of exact multi-triplon bound states, which are the key to the unconventional thermodynamic and dynamical response of the frustrated ladder and allow a complete analytical interpretation of our numerical results.

A direct approach to the evaluation of Eq. (3) is based on full diagonalization, i.e., on the computation of all eigenvalues, $E_{i}$, and the corresponding eigenstates, $|i\rangle$. Although our initial computations for ladders of $10 \times 2$ spins were performed on the high-performance computers of HLRN II, this calculation is actually feasible on a modern desktop. The primary bottleneck is the requirement for approximately 470 GBytes of hard-disk space to store all the necessary matrix elements, $\left\langle j\left|S^{z}(\vec{k})\right| i\right\rangle$, for each set of exchange constants.

To proceed to larger systems, and here we present data for ladders up to $16 \times 2$ spin- $1 / 2$ sites, it is no longer possible to obtain the full spectrum. However, one may truncate the sum over $i$ in Eq. (3) to low energies $E_{i}$ and use the Lanczos algorithm $[84,85]$ to compute these low-lying states; the spectral sum over $j$ is then evaluated by a continued-fraction expansion for each eigenstate $|i\rangle$ [85-87]. We retain at least 20 initial states, $|i\rangle$, in each symmetry sector for ladders of $L=14$ 
rungs, pushing this up to several hundred per symmetry sector for $L=12$. The truncation to low energies restricts the validity of this approximation to low temperatures. Thus, although we have access only to smaller system sizes ( $L=10$ rungs) at higher temperatures, the finite-size effects we wish to gauge are important primarily at lower temperatures. For this reason, we have pushed our calculations to the largest combinations of system size and temperature allowed by our computational resources.

One specific advantage of ED is that we have access to the weight of individual poles of the spectral function, i.e., the coefficients of the individual $\delta$ functions in the limit $\eta=0$. In the context of full diagonalization, this is contained in the spectral representation [Eq. (3)], while in the continued-fraction expansion these weights are obtained from the eigenvectors of the associated tridiagonal Lanczos matrix [85].

The effects of the finite temperature are incorporated through Boltzmann weighting factors $e^{-E_{i} / T}$ on every energy level $E_{i}$ in the spectrum. We have performed calculations for ladders of $L=6,8,10,12,14$, and 16 rungs at zero temperature, and up to $L=14$ at finite temperatures, in order to analyze the effects of finite system size. We will demonstrate that these effects are in general very limited, because of the extremely short correlation length of the maximally frustrated system, and it is only arbitrarily close to $j^{\prime}=j_{c}^{\prime}$ (in fact where multi-triplon bound states with $n \approx L$ become relevant) that they become visible in the dynamical response.

The two-leg ladder has two types of spectral function, those symmetric or antisymmetric between the legs, corresponding as above to the quantum numbers $k_{\perp}=0$ and $\pi$, and we will show both. At $j^{\prime}>j_{c}^{\prime}$ (rung-singlet ground state), processes changing the number of triplons, $l$, by an odd number appear in the antisymmetric channel, while those involving even numbers (including zero, as in intramultiplet transitions) are symmetric. The selection rules of a neutron-scattering process, and indeed of the matrix elements in Eq. (3), are that the change in total spin may be only $\Delta S=0, \pm 1$.

For the fully frustrated ladder, these rules combine with the conservation law on the total spin of each rung [49] to dictate that direct processes are allowed only from sectors with $l$ excited rung triplons to sectors with $l$ or $l \pm 1$. At zero temperature, the rung-singlet ground state may therefore be excited by the antisymmetric operators of Eq. (3) only into the antisymmetric channel, and we stress that it is not possible to couple directly to the triplet branch of an $n$-triplon bound state with $n \geqslant 2$. Because the rung spin is a good quantum number for all parameters, the same is true for the rung-triplet ground state. This result is a consequence of the perfect frustration and can be relaxed if the frustration is incomplete, albeit with small matrix elements, as we will illustrate in Secs. III C and IV.

As a consequence, all excitation processes of the fully frustrated ladder to sectors of higher $l$ take place only at finite temperatures, where the probability for multi-triplon states to be populated is nonvanishing. However, the transitions between these states and others within the thermal population remain restricted to those obeying the selection rules $\Delta S=$ $0, \pm 1$ and $\Delta l=0, \pm 1$. The effect of the temperature is to alter the relative weights of the different terms in Eq. (3), establishing the thermal evolution of $S(\vec{k}, \omega)$.

\section{B. Fully frustrated ladders}

We first provide results to illustrate the evolution of the dynamical spectral function with temperature. Our primary focus is on the fully frustrated ladder $\left(J_{\|}=J_{\times}=1\right)$, for which we compare the situations within and beyond the exact bound-state regime $\left(j^{\prime}>j_{c}^{\prime}\right.$ and $\left.j^{\prime}<j_{c}^{\prime}\right)$, with particular attention paid to the critical region around $j^{\prime}=j_{c}^{\prime}$. To gain more perspective on these results, we also compare the fully frustrated case with unfrustrated $\left(J_{\times}=0\right)$ and less frustrated ladders $\left(J_{\times}=0.9 J_{\|}\right)$.

\section{1. $j^{\prime}>j_{c}^{\prime}$}

We begin by presenting the calculated dynamical structure factor for a fully frustrated ladder with $J_{\perp}=2$ and $J_{\|}=$ $J_{\times}=1$. Figure 3 shows the spectral weights for a range of temperatures between 0 and $J_{\perp}$, with the symmetric channel in the left panels and the antisymmetric channel on the right. We draw attention to the fact that the color contours in all the figures in this section represent logarithmic intensity scales, and thus the strong branches are indeed features of very high relative weight. At low temperatures, only one feature is visible, the one-triplon excitation appearing in the antisymmetric channel. Its band dispersion, $\omega(k)=J_{\perp}$, is completely flat as a consequence of the perfect frustration. As the temperature increases, increasing spectral weight may be found in this channel at a significant number of different, discrete, and nondispersive energy levels, which must arise from two- or more-triplon excitations. The number of visible levels continues to increase with $T$, as, with one obvious exception, does the weight they contain. The one-triplon mode has clearly lost appreciable spectral weight once $T=0.5 J_{\perp}$, and this loss continues with $T$.

In the symmetric channel, we also find a range of discrete, nondispersive excitations, but these have zero spectral weight at $k_{\|}=0$ and a maximum at $k_{\|}=\pi$, causing a modulation of the scattered intensity. Again, both the number of visible levels and their weight increase with temperature. An explanation for the origin, possible degeneracy, and intensity evolution of the strongest energy levels is deferred to Sec. IV.

\section{2. $j^{\prime}<j_{c}^{\prime}$}

Remaining with fully frustrated ladders, in Fig. 4, we show similar results for a ladder with $J_{\perp}=J_{\|}=J_{\times}=1$, values for which the system is located in the rung-triplet (Haldane-type) phase. The antisymmetric channel again shows completely nondispersive triplet bands at zero temperature, but in this case more than one. As the temperature is increased, these are joined by a larger number of weaker levels, which have almost merged into an intensity continuum when $T=J_{\perp}$. We observe that the one-triplon level dominating the low- $T$ spectrum has vanished almost completely at this temperature.

In the symmetric channel, at low temperatures, one may recognize the characteristic triplet ("magnon") dispersion relation of the Haldane chain, whose dynamical structure factor at $T=0$ is shown in Fig. 4(b) of Ref. [88] for similar 


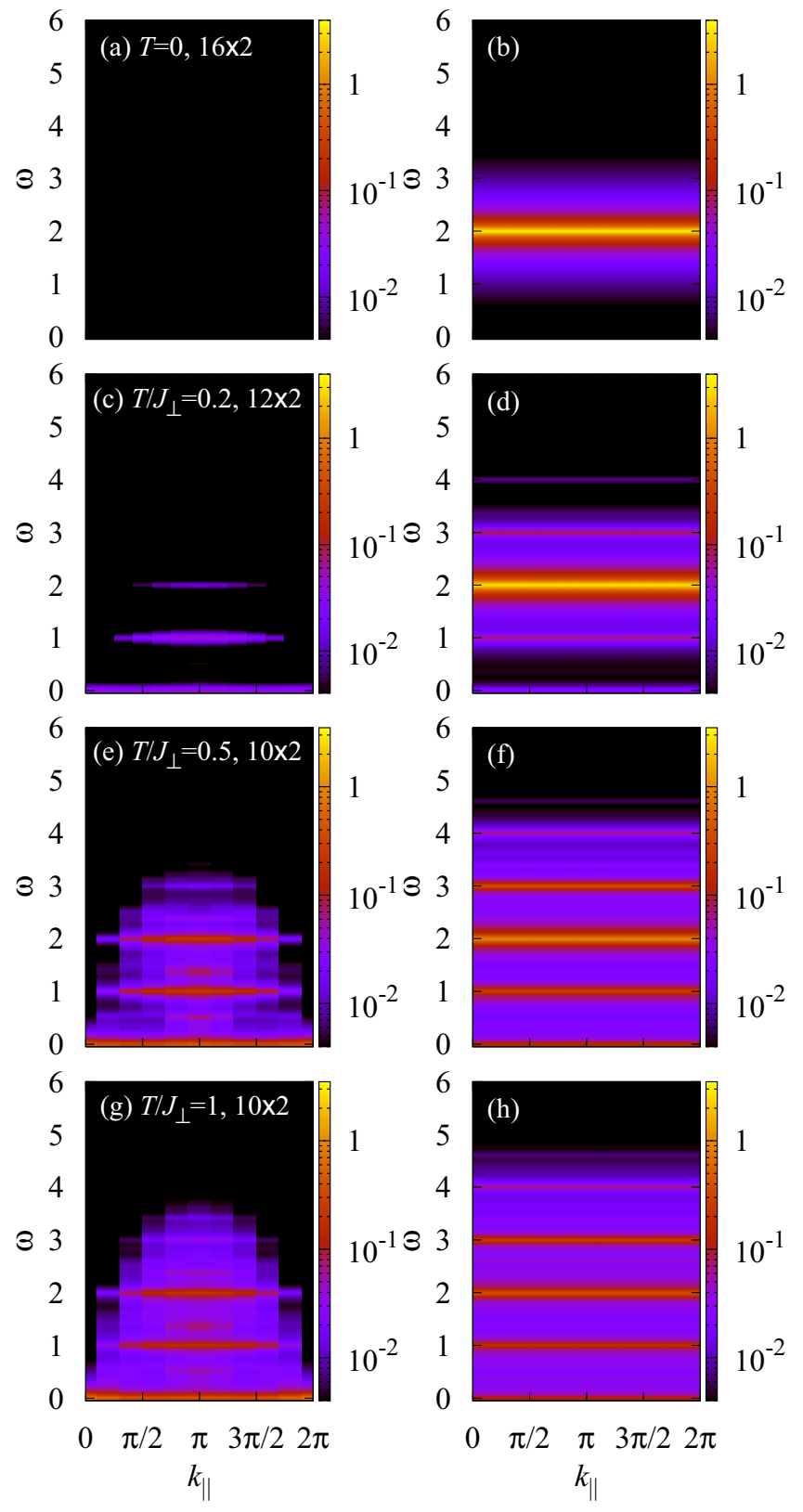

FIG. 3. Dynamical structure factor in (a), (c), (e), and (g) the symmetric and (b), (d), (f), and (h) the antisymmetric channel for the ladder of Fig. 1 with coupling parameters $J_{\perp}=2$ and $J_{\|}=J_{\times}=1$, for temperatures of (a) and (b) $T / J_{\perp}=0(16 \times 2$ spins), (c) and (d) 0.2 ( $12 \times 2$ spins), (e) and (f) 0.5 , and (g) and (h) 1 . The system size for $T=0.5$ and 1 is $10 \times 2$ spins and a Lorentzian broadening $\eta=J_{\|} / 20$ is applied to the data.

system sizes. More specifically, in Fig. 4(a), we compute a band minimum at $k_{\|}=\pi$ of $\Delta / J_{\|} \simeq 0.443$ for the $L=16$ case [52,89], compared with the true Haldane gap of $\Delta / J_{\|}=$ 0.4105 , and find the band maximum where it is cut off by the descent of the two-magnon continuum towards $k_{\|}=0$ and $2 \pi$ [90]. At intermediate temperatures, more flat-band features begin to appear in the spectral weight, and at high temperatures the weight distribution is quite similar to the case with $J_{\perp}=2$, shown in Fig. 3, suggesting the predominance of local physics.

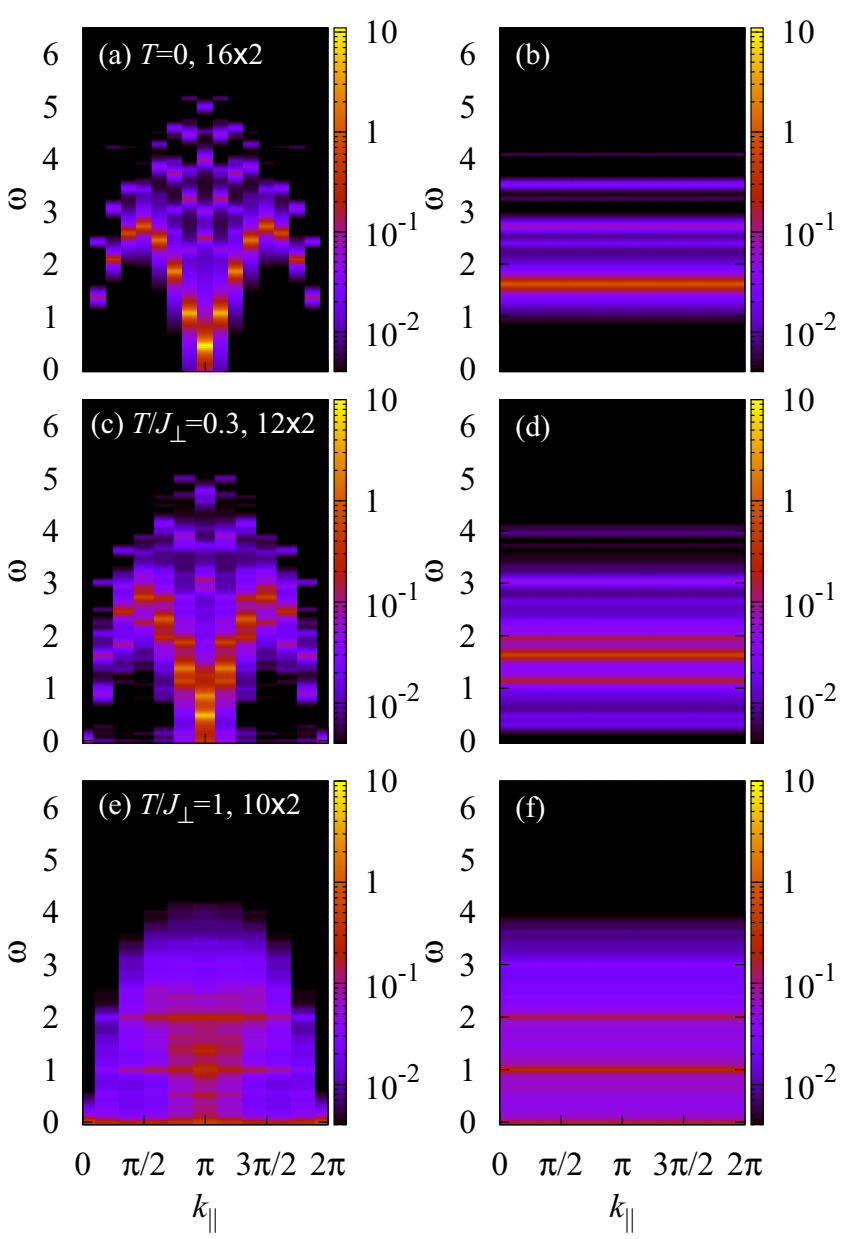

FIG. 4. Dynamical structure factor in (a), (c), and (e) the symmetric and (b), (d), and (f) the antisymmetric channel for the ladder of Fig. 1 with coupling parameters $J_{\perp}=J_{\|}=J_{\times}=1$, for temperatures of (a) and (b) $T / J_{\perp}=0(16 \times 2$ spins), (c) and (d) 0.3 $(12 \times 2$ spins $)$, and (e) and (f) $1(10 \times 2$ spins). A Lorentzian broadening $\eta=J_{\|} / 20$ is applied to the data.

\section{3. $j^{\prime} \rightarrow j_{c}^{\prime}$}

As discussed in Sec. II and demonstrated in the thermodynamic properties of the system [34], the most dramatic phenomena in the fully frustrated ladder occur close to the quantum phase transition $\left(j_{c}^{\prime}\right)$, where large numbers of multitriplet bound states appear at very low energies. However, these are of necessity extended objects, and therefore our calculations for small systems are no longer perfectly representative of the thermodynamic limit as $j^{\prime} \rightarrow j_{c}^{\prime}$. Nevertheless, in Fig. 5 we show $S^{z z}(k, \omega, T)$ calculated for a ladder with $J_{\perp}=1.45$ and $J_{\|}=J_{\times}=1$, where we find our results to be largely converged at $L=14$; thus the critical regime dominated by truly high- $n$ bound states remains rather narrow [34]. It is clear that the spectral weight evolves with temperature in a manner very similar to the $J_{\perp}=2$ case (Fig. 3), which may be regarded as representing generic and noncritical fully frustrated behavior, but in an accelerated manner. The weight growth visible in Fig. 5 is equivalent to an effective rescaling of the temperature by a factor of approximately 2.5 compared with Fig. 3 , and yet 


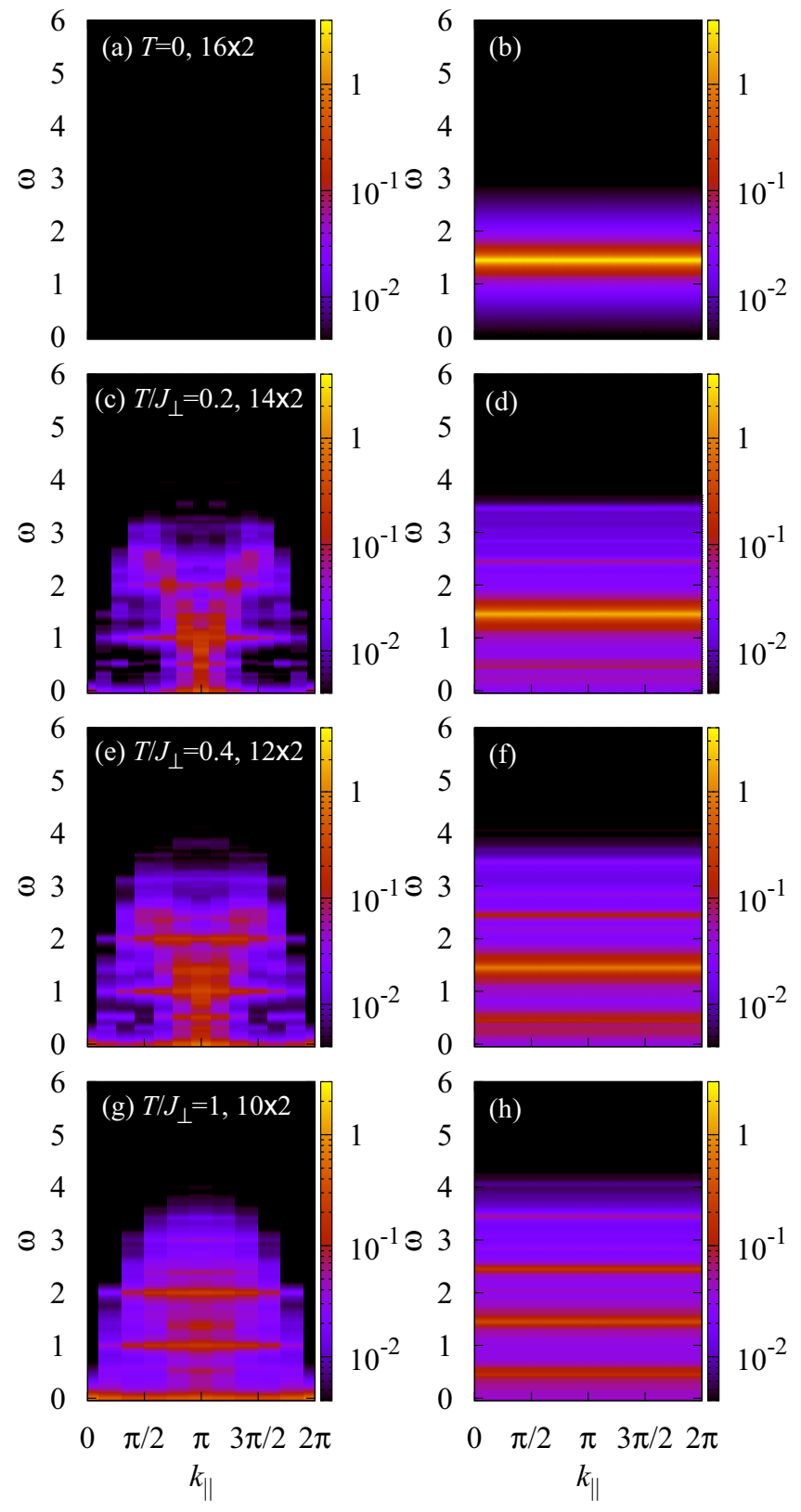

FIG. 5. Dynamical structure factor in (a), (c), (e), and (g) the symmetric and (b), (d), (f), and (h) the antisymmetric channel for the ladder of Fig. 1 with coupling parameters $J_{\perp}=1.45$ and $J_{\|}=J_{\times}=$ 1 , for temperatures of (a) and (b) $T / J_{\perp}=0$ (16×2 spins), (c) and (d) 0.2 (14×2 spins), (e) and (f) 0.4 ( $12 \times 2$ spins), and (g) and (h) 1 ( $10 \times 2$ spins). A Lorentzian broadening $\eta=J_{\|} / 20$ is applied to the data.

was obtained with no rescaling of energies (although we note that $T$ is normalized to $J_{\perp}$ ).

To summarize our observations for fully frustrated ladders in the rung-singlet regime, the dynamical spectral function is dominated by discrete and nondispersive excitations, whose origin lies in the existence of exact bound states. As a function of temperature, all of the bound-state levels are populated increasingly at the cost of the intensity in the one-triplon band. The intensity distribution is $k_{\|}$-independent in the antisymmetric channel, but peaked at $k_{\|}=\pi$ and vanishing at $k_{\|}=0$ in the symmetric channel. The dependence of this thermal evolution effect on the inter-rung coupling ratio may be broadly characterized, from studies we do not show here for reasons of space, as follows. The dynamical spectral function for ladders with values of $j^{\prime} \gtrsim 3$ varies little from the case $j^{\prime}=\infty$ (isolated rung singlets) for any realistic values of the temperature. For values $1.5 \lesssim j^{\prime} \lesssim 3$, we observe the essential phenomenon of thermal evolution in a frustrated system, that spectral weight from the one-triplon band is redistributed to lower and higher energies by the formation of few-triplon bound states. For values $j_{c}^{\prime} \lesssim j \leqslant 1.5$, we find highly anomalous versions of this effect, with extremely rapid spectral-weight transfer out of the one-triplon band and into bound states of many different triplons. For values $j<j_{c}^{\prime}$, we find a composite spectral function containing some features of the Haldane chain [90] and some of local excitations (rung-singlet bound states), a topic we discuss in more detail in Sec. IV.

\section{Unfrustrated and partially frustrated ladders}

To benchmark the effects of frustration on the temperature dependence of the dynamical spectral function, we switch them off in whole or in part. In Fig. 6, we show results analogous to those of Fig. 3, but for an unfrustrated 14-rung ladder with $J_{\perp}=2, J_{\|}=1$, and $J_{\times}=0$. In this case, the one-triplon band visible in the low-temperature antisymmetric response has a clear dispersion, with its minimum $\left(\omega / J_{\|} \approx 1\right)$ at $k_{\|}=\pi$ and a band width of approximately $2 J_{\|}$. This band is broadened into a continuum of available states as a function of increasing temperature, and at $T \approx J_{\perp}$ it has a width of order $J_{\perp}$, as expected for conventional thermal broadening. The low-temperature spectrum in the symmetric channel is that of a dispersive two-triplon bound state [91-94], which has a strongly $k_{\|}$-dependent intensity and a loss of weight from the high-energy feature reappearing at low energies for higher temperatures. In the thermodynamic limit, these bands are continuous, and simply broaden at finite temperatures into a continuum of available states with equal weights.

The characteristic features arising from the discrete energy spectrum of the fully frustrated case can be recovered if the frustration parameter, $J_{\times}$, is increased continuously from 0 to 1 . The conventional spectral features of Fig. 6 are changed smoothly into the very different form of Fig. 3 by the emergence of preferred, discrete levels from the continua, whose widths decrease systematically to very narrow values, and whose dispersion decreases until the bands become completely flat. This evolution is represented in Fig. 7 for the case $J_{\times}=0.9$. The frustration in this case is strong, and the bands only weakly dispersive, such that the fingerprints of multi-triplon bound states emerge with increasing temperature. However, the continuum nature of the bands remains evident in the two-magnon response at $T=0$ in the symmetric channel, as well as in the continuous nature of the thermal broadening in the antisymmetric channel at $T / J_{\perp}=1$, which on close inspection lacks the weakly varying intensity bands visible between the primary modes in Figs. 3 and 5. 


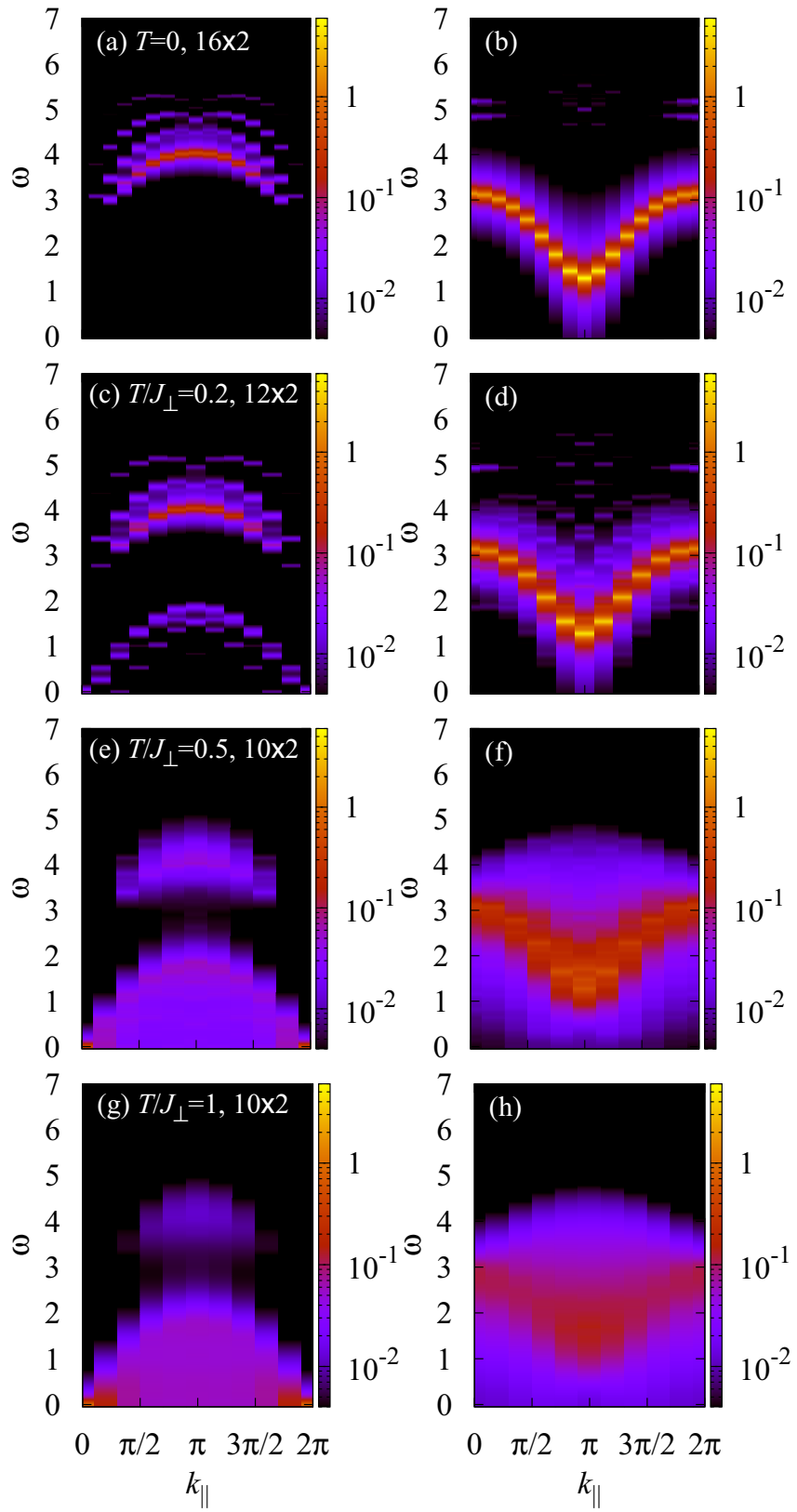

FIG. 6. Dynamical structure factor in (a), (c), (e), and (g) the symmetric and (b), (d), (f), and (h) the antisymmetric channel for the ladder of Fig. 1 with coupling parameters $J_{\perp}=2, J_{\|}=1$, and $J_{\times}=0$, for temperatures of (a) and (b) $T / J_{\perp}=0(16 \times 2$ spins), (c) and (d) $0.2(12 \times 2$ spins), (e) and (f) 0.5 , and (g) and (h) 1 (both $10 \times 2$ spins). A Lorentzian broadening $\eta=J_{\|} / 20$ is applied to the data.

\section{Infinite-temperature spectral functions}

One of the most striking features of the spectra we calculate is their behavior at very high temperatures. Figure 8 shows the dynamical spectral functions for all the ladders of Figs. 3 to 7 as $T \rightarrow \infty$. The conventional expectation is the situation illustrated in Figs. 8(g) and 8(h) for the unfrustrated ladder, where the spectral weight is spread uniformly to all available energies. By contrast, the primary spectral features of the fully frustrated ladders [Figs. 8(a)-8(f)] not only persist at

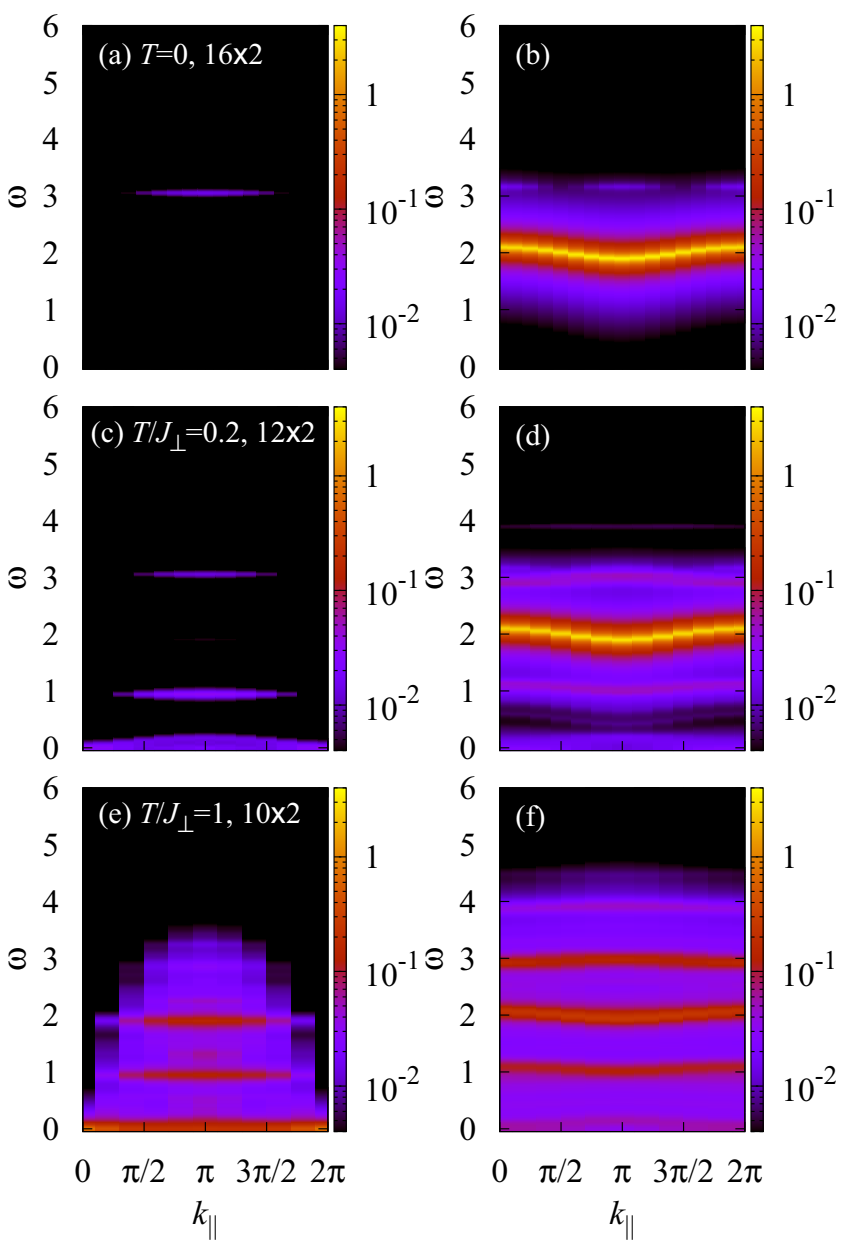

FIG. 7. Dynamical structure factor in (a), (c), and (e) the symmetric and (b), (d), and (f) the antisymmetric channel for the ladder of Fig. 1 with coupling parameters $J_{\perp}=2, J_{\|}=1$, and $J_{\times}=0.9$, for temperatures of (a) and (b) $T / J_{\perp}=0$ (16×2 spins), (c) and (d) 0.2 $(12 \times 2$ spins $)$, and (e) and (f) $1(10 \times 2$ spins $)$. A Lorentzian broadening $\eta=J_{\|} / 20$ is applied to the data.

infinite temperature but remain completely sharp. It is clear that the discrete support, by which is meant the $T=0$ spectrum of nondispersive bound states, leads to preferred scattering processes at these specific energies at all temperatures. This perfect discreteness is the consequence of the perfect frustration, and in the nearly-frustrated ladder [Figs. 8(i) and $8(\mathrm{j})$ ] one observes that the sharpness begins to be lost, although the levels do persist; we examine both effects in more detail in Sec. IV.

\section{ANALYSIS OF SPECTRAL WEIGHTS}

We begin a quantitative discussion of our results for the dynamical structure factor by displaying our intensity data (Figs. 3 to 8 ) as functions of energy for one specific wave vector, taken to be $k_{\|}=\pi$; although the intensities are identical for all wave vectors in the antisymmetric channel, this is not the case in the symmetric channel. Again these data are most transparent on a logarithmic scale and Figs. 9 to 13 demonstrate clearly the increasing importance of increasing numbers of 

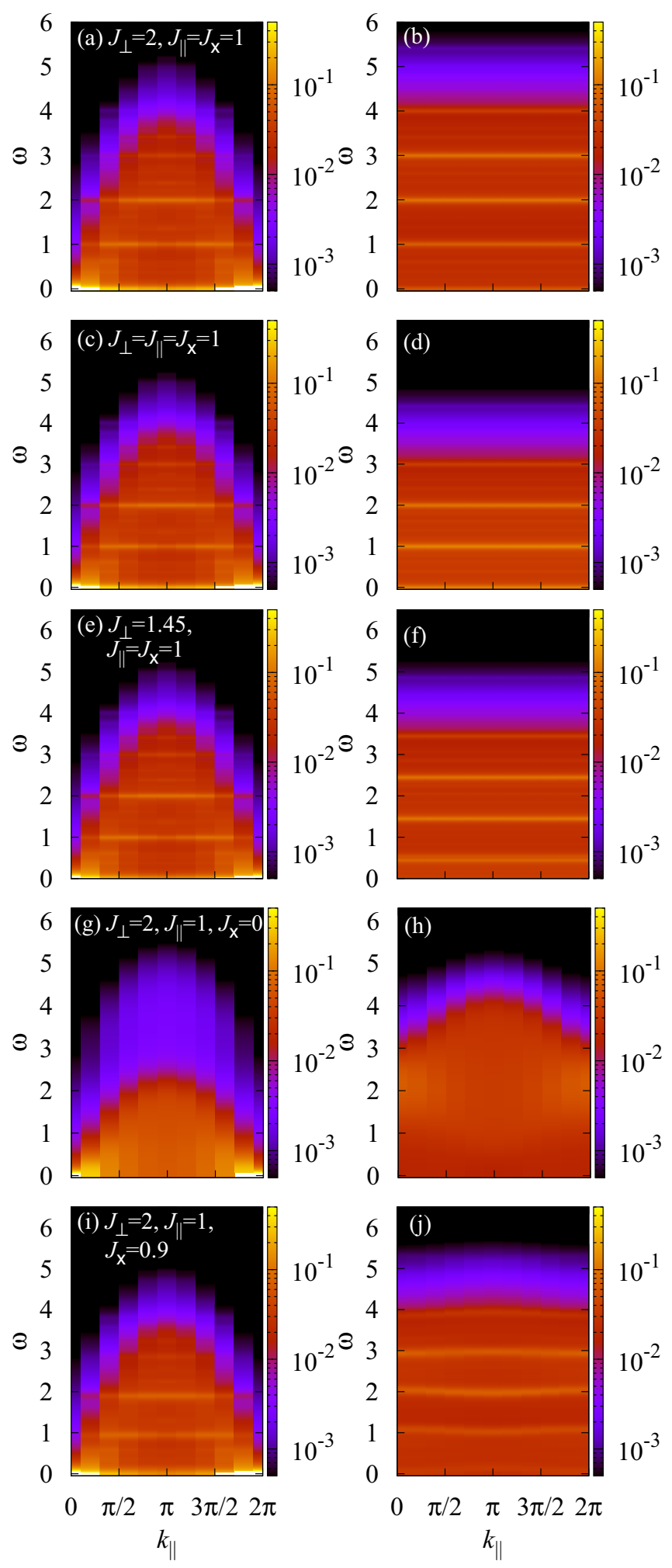

FIG. 8. Dynamical structure factor in (a), (c), (e), (g), and (i) the symmetric and (b), (d), (f), (h), and (j) the antisymmetric channel at infinite temperature for ladders with coupling parameters $J_{\perp}=2$ and $J_{\|}=J_{\times}=1$ (a) and (b); $J_{\perp}=J_{\|}=J_{\times}=1$ (c) and (d); $J_{\perp}=1.45$ and $J_{\|}=J_{\times}=1(\mathrm{e})$ and (f); $J_{\perp}=2, J_{\|}=1$, and $J_{\times}=0(\mathrm{~g})$ and (h); $J_{\perp}=2, J_{\|}=1$, and $J_{\times}=0.9$ (i) and (j). The system size is $10 \times 2$ spins throughout this figure and a Lorentzian broadening $\eta=J_{\|} / 20$ is applied to the data. bound-state energy levels as the temperature is raised. In every panel, we show data obtained for three successive system sizes in order to gauge their finite-size effects. The lines display data obtained with a broadening factor of $\eta=0.05 J_{\|}$, and we comment that, as a result of the logarithmic intensity scale, the entire line shape visible in the figures of this section is a consequence of $\eta$.

The primary features of the dynamical spectral functions for which we seek a quantitative explanation are (i) the nature of the processes generating the discrete spectra of the fully frustrated ladder, which we address in Sec. IV A, and (ii) the origin of the rapid transfer of spectral weight, which we discuss in Secs. IV B and IV C. We first summarize the qualitative features of the results we show for the two different types of ground state, namely the rung-singlet phase of Figs. 9 and 11 and the rung-triplet (Haldane) phase of Fig. 10. Although it stands to reason that there are different types of excited state for $j^{\prime}>j_{c}^{\prime}$ and $j^{\prime}<j_{c}^{\prime}$, the lower-energy states of one phase are higher-energy excitations of the other, and thus both sides of the transition contain messages for interpreting the opposite one.

In the rung-singlet phase shown in Fig. 9, we comment first that very few finite-size effects (discrepancies between the curves obtained for the three system sizes shown) are visible, indicating that even these short systems capture all of the physics away from $j_{c}^{\prime}$. The one-triplon peak in the antisymmetric channel loses weight systematically as a function of temperature to a small number of dominant and, for this parameter choice, symmetrically distributed states lying above a rising background. The response in the symmetric channel is strongest at zero energy, and shows weaker finite-temperature contributions from a greater number of levels. Figure 11 shows the same physics developing at lower temperatures, as well as additional peaks appearing due to the deliberate asymmetry of the parameters (Sec. IV A).

The spectrum of the rung-triplet phase $\left(j^{\prime}<j_{c}^{\prime}\right.$, Fig. 10) shows the predominance of weight in the symmetric channel, and displays significant finite-size effects. These are expected in general if the origin of the features lies in the "Haldane" part of the spectrum, which contains long-wavelength excitations, but not from the localized (bound-state) features. However, we comment that the strongest finite-size effects in Fig. 10(a) actually appear in the three-particle continuum [90], whereas those in the one-triplon line are small. The loss of strong finite-size features at higher temperatures implies that the primary contributions in this regime are in fact from few-rung processes; here one may borrow from the $j^{\prime}>j_{c}^{\prime}$ phase to interpret these as isolated rung singlets, and (nonexact) bound states of rung singlets, in a background of rung triplets. Similarly, as noted in Sec. II, the interpretation of the spectral function at $j^{\prime}>j_{c}^{\prime}$ can also be borrowed from the $j<j_{c}^{\prime}$ side in the form of open $n$-site Haldane chains separated by singlet rungs. The presence of such segmented Haldane chains is clearest in the symmetric channel, in the shape of the envelope of spectral weight in Figs. 3 to 5 .

The physics of the spectral-weight distribution, and of its thermal redistribution, is shown most clearly in Fig. 5 for a system close to $j_{c}^{\prime}$. The intensities of the discrete energy levels over the entire spectrum rise rapidly with temperature, and 

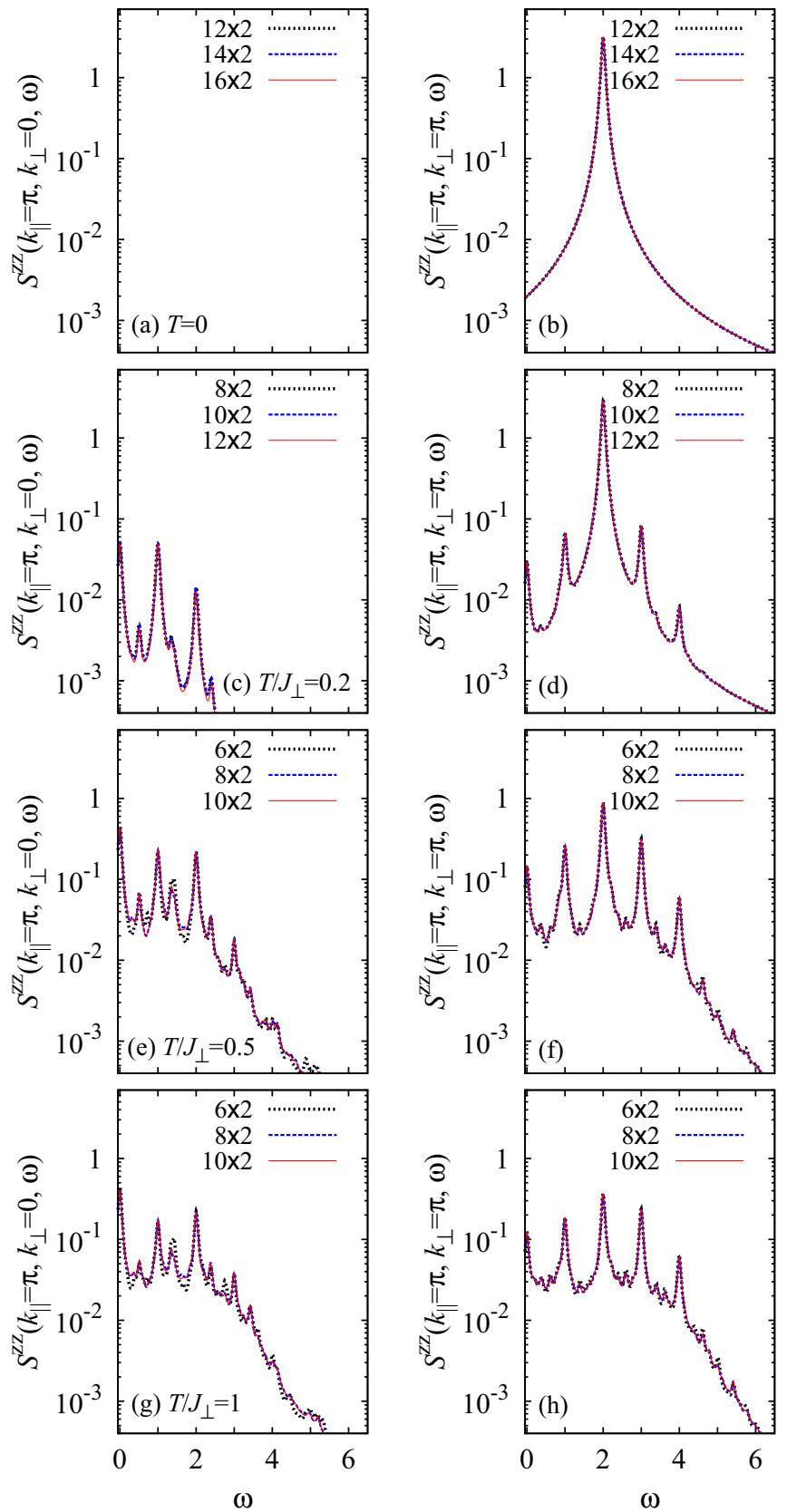

FIG. 9. Intensity at $k_{\|}=\pi$ in (a), (c), (e), and (g) the symmetric and (b), (d), (f), and (h) the antisymmetric channel, shown as a function of energy for the ladder of Fig. 1 with the system size specified, coupling parameters $J_{\perp}=2$ and $J_{\|}=J_{\times}=1$, and $\eta=J_{\|} / 20$, for temperatures of (a) and (b) $T / J_{\perp}=0$, (c) and (d) 0.2 , (e) and (f) 0.5 , and (g) and (h) 1 .

the concomitant fall in the intensity of the one-triplon band is quantified in Fig. 11. The redistribution effect among discrete levels shown in this figure provides a stark contrast with the unfrustrated ladder, whose intensity at $k_{\|}=\pi$ is shown in Fig. 12. For the minimum of the dispersive ladder bands, one observes a dominant low-lying peak in the antisymmetric channel spreading nonuniformly to all energies with increasing temperature, whereas the symmetric channel preserves distinct but dispersive low- and high-energy contributions to significant
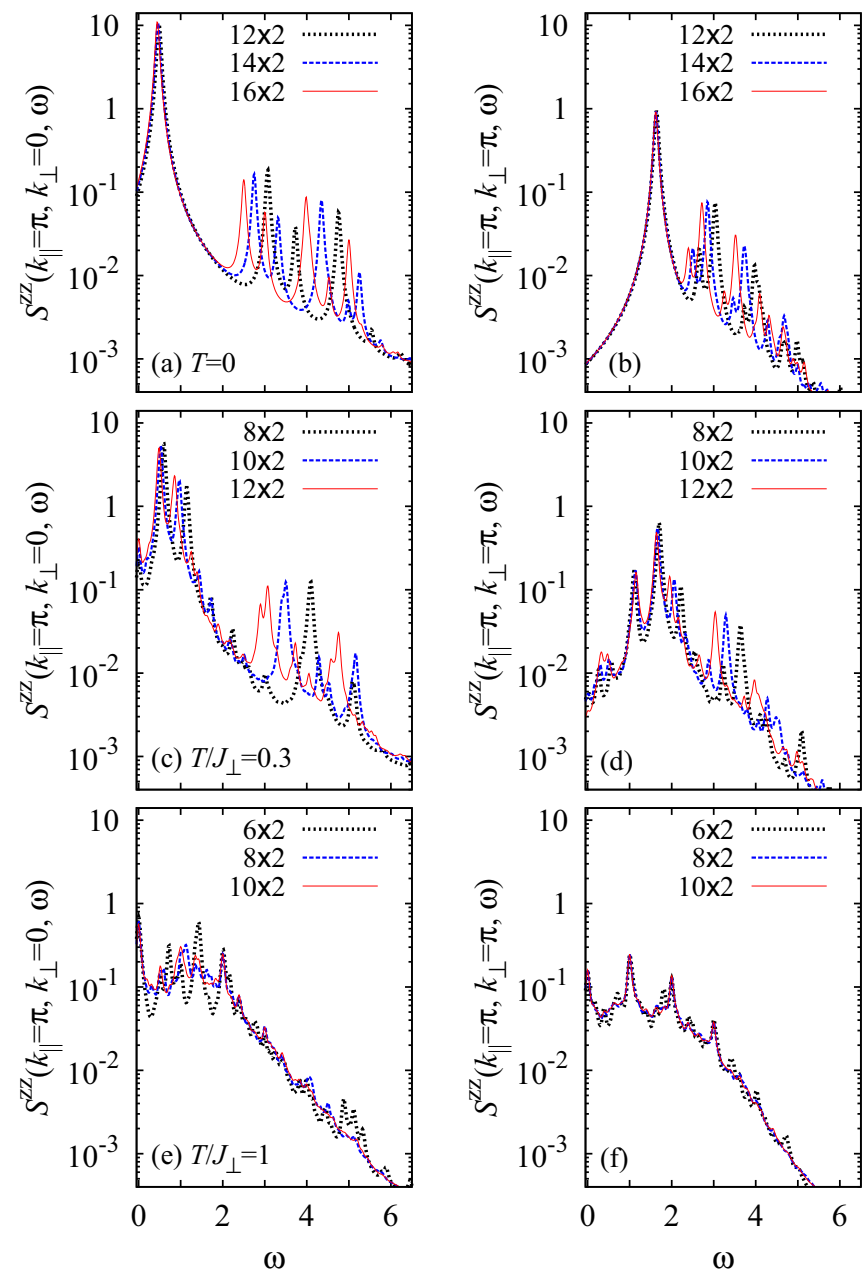

FIG. 10. Intensity at $k_{\|}=\pi$ in (a), (c), and (e) the symmetric and (b), (d), and (f) the antisymmetric channel, shown as a function of energy for the ladder of Fig. 1 with the system size specified, coupling parameters $J_{\perp}=J_{\|}=J_{\times}=1$, and $\eta=J_{\|} / 20$, for temperatures of (a) and (b) $T / J_{\perp}=0$, (c) and (d) 0.3 , and (e) and (f) 1 .

temperatures; the dominant low-temperature feature is the two-triplon bound state mentioned in Sec. III C [91-94].

Figure 13 provides the imperfectly frustrated hybrid of the two different types of response. The perfect frustration therefore produces a very unusual but characteristic spectrum of discrete, flat bands, which are determined by the bound states discussed in Sec. II; the fact that they appear in the spectral function with a very specific hierarchy of spectral weights indicates that their ratios are determined not only by the temperature but also by specific matrix elements.

\section{A. Excitation processes}

To identify the excitation processes present in the calculated spectra, we use the energies of the known bound states and begin with the smallest clusters. The results for the spectra of the $n=2$ and 3 multiplets are given in Appendix A. Our choice of parameters in all the figures of Secs. III and IV for fully frustrated ladders is such that $J_{\|}=J_{\times}=1$, with only $J_{\perp}$ changing the ratio $j^{\prime}$. Because $J_{\|}$determines the multiplet splitting of the bound states, and all level separations 

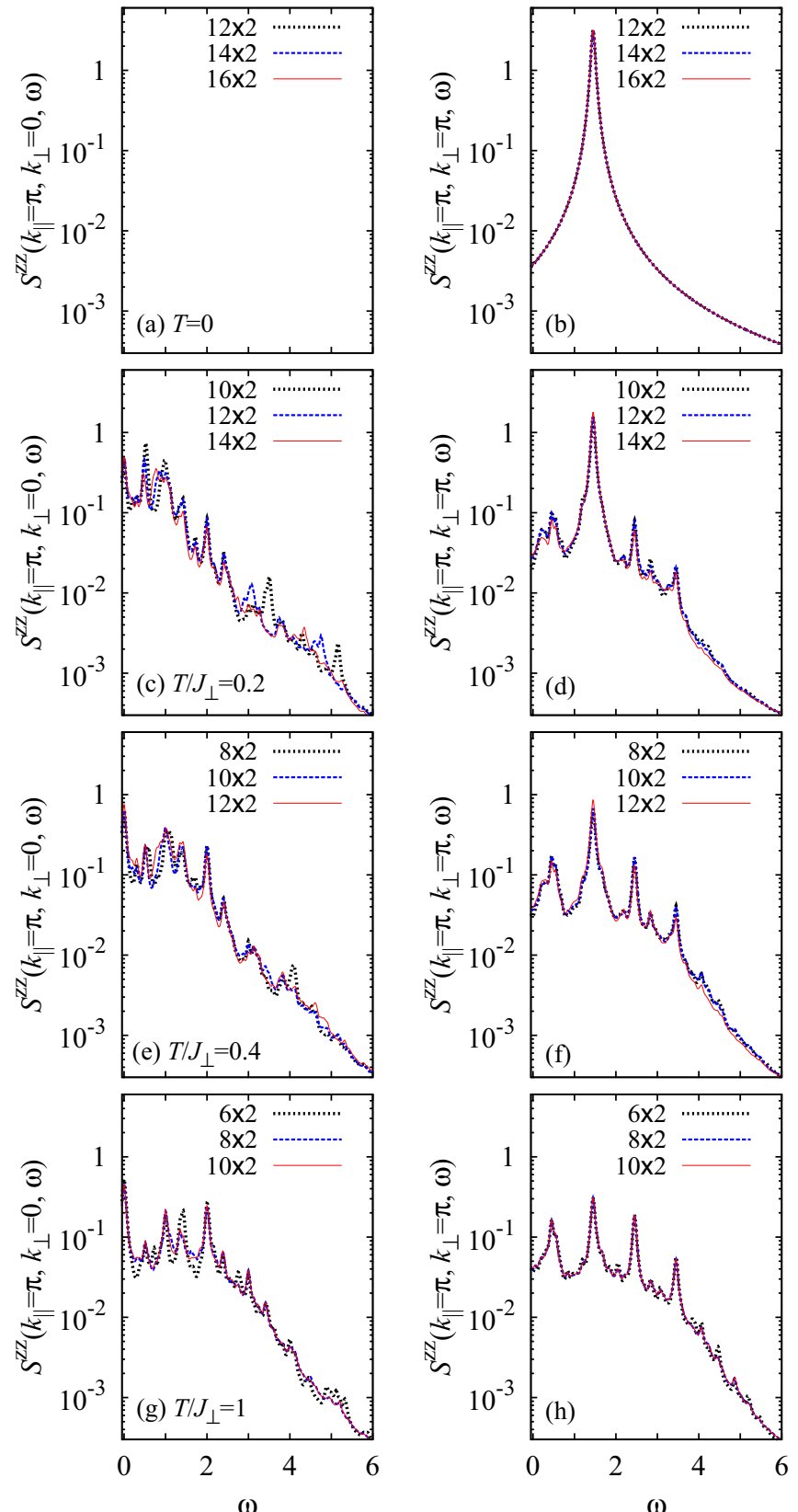

FIG. 11. Intensity at $k_{\|}=\pi$ in (a), (c), (e), and (g) the symmetric and (b), (d), (f), and (h) the antisymmetric channel, shown as a function of energy for the ladder of Fig. 1 with the system size specified, coupling parameters $J_{\perp}=1.45$ and $J_{\|}=J_{\times}=1$, and $\eta=J_{\|} / 20$, for temperatures of (a) and (b) $T / J_{\perp}=0$, (c) and (d) 0.2 , (e) and (f) 0.4 , and (g) and (h) 1 .

are integral for $n=2$ and 3 , any excitations with irrational energies must automatically be a consequence of transitions involving clusters with $n=4$ or higher. However, this situation has the twin disadvantages that primary $n=2$ and 3 levels are always degenerate and that "commensurate" values of $J_{\perp} / J_{\|}$ can cause additional energetic degeneracies between different excitation processes.

Considering only the antisymmetric channel, the onetriplon excitation is located at an energy of $j^{\prime}$ (in units of $J_{\|}$) and the leading bound-state energies in Figs. 3 (9) and 5 (11)
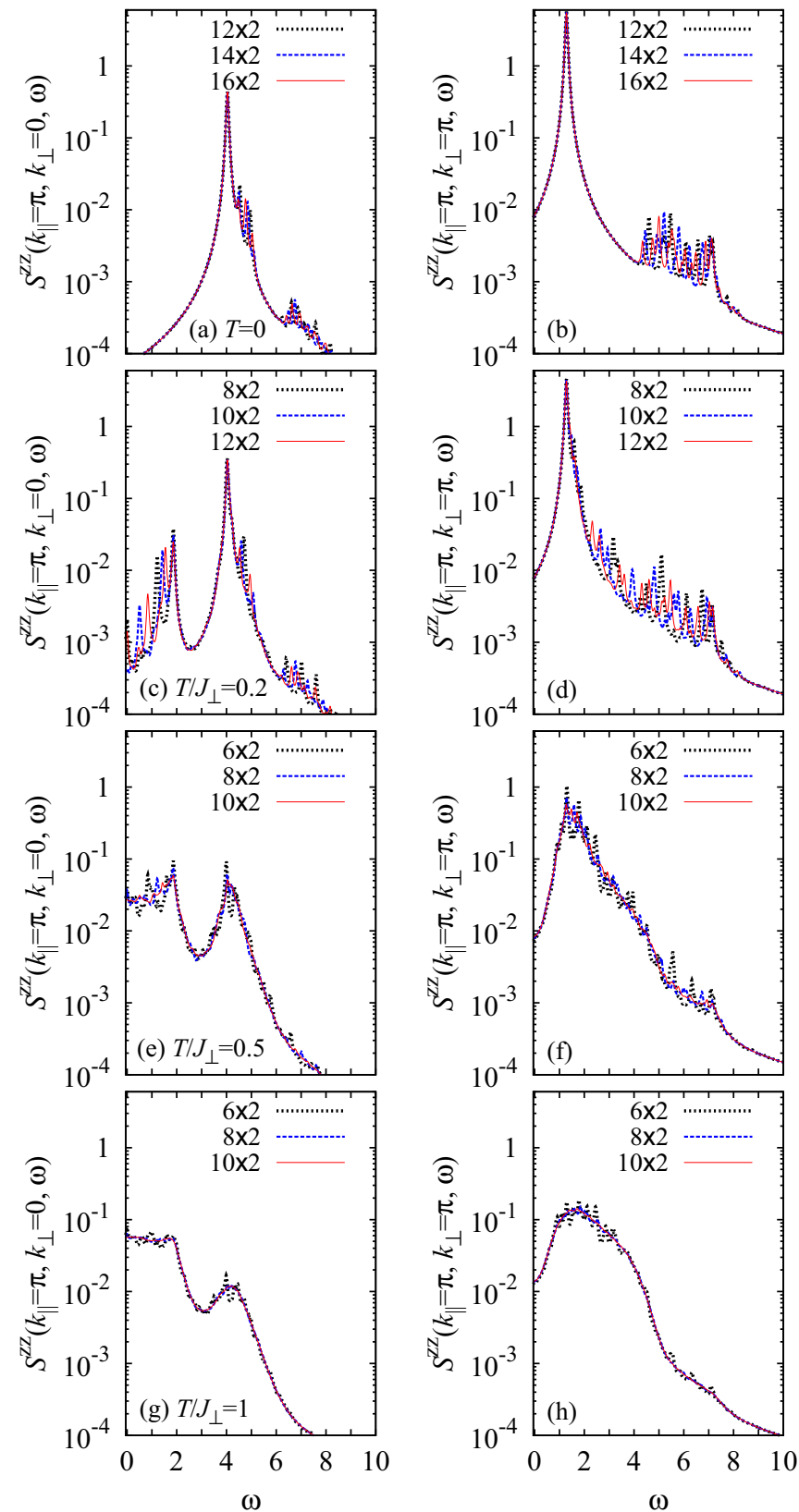

FIG. 12. Intensity at $k_{\|}=\pi$ in (a), (c), (e), and (g) the symmetric and (b), (d), (f), and (h) the antisymmetric channel, shown as a function of energy for the ladder of Fig. 1 with the system size specified, coupling parameters $J_{\perp}=2, J_{\|}=1$, and $J_{\times}=0$, and $\eta=J_{\|} / 20$, for temperatures of (a) and (b) $T / J_{\perp}=0$, (c) and (d) 0.2 , (e) and (f) 0.5 , and (g) and (h) 1 .

are located at $\left|j^{\prime}-2\right|, j^{\prime}-1$, and $j^{\prime}+1$. These all arise from transitions between the one-triplon state and the twotriplon bound state (for which we adopt the notation $1 \rightarrow 2$ ), respectively its singlet, triplet, and quintet components. We note (Appendix A) that there is no selection rule prohibiting excitation processes from a triplet to a triplet in the multiplet basis. We find that the first process is degenerate with the one-triplon band for the case $j^{\prime}=2$ and thus we use "incommensurate" values of $j^{\prime}$ to isolate the individual contributions from every process where possible. For the choice $j^{\prime}=1.45$, 

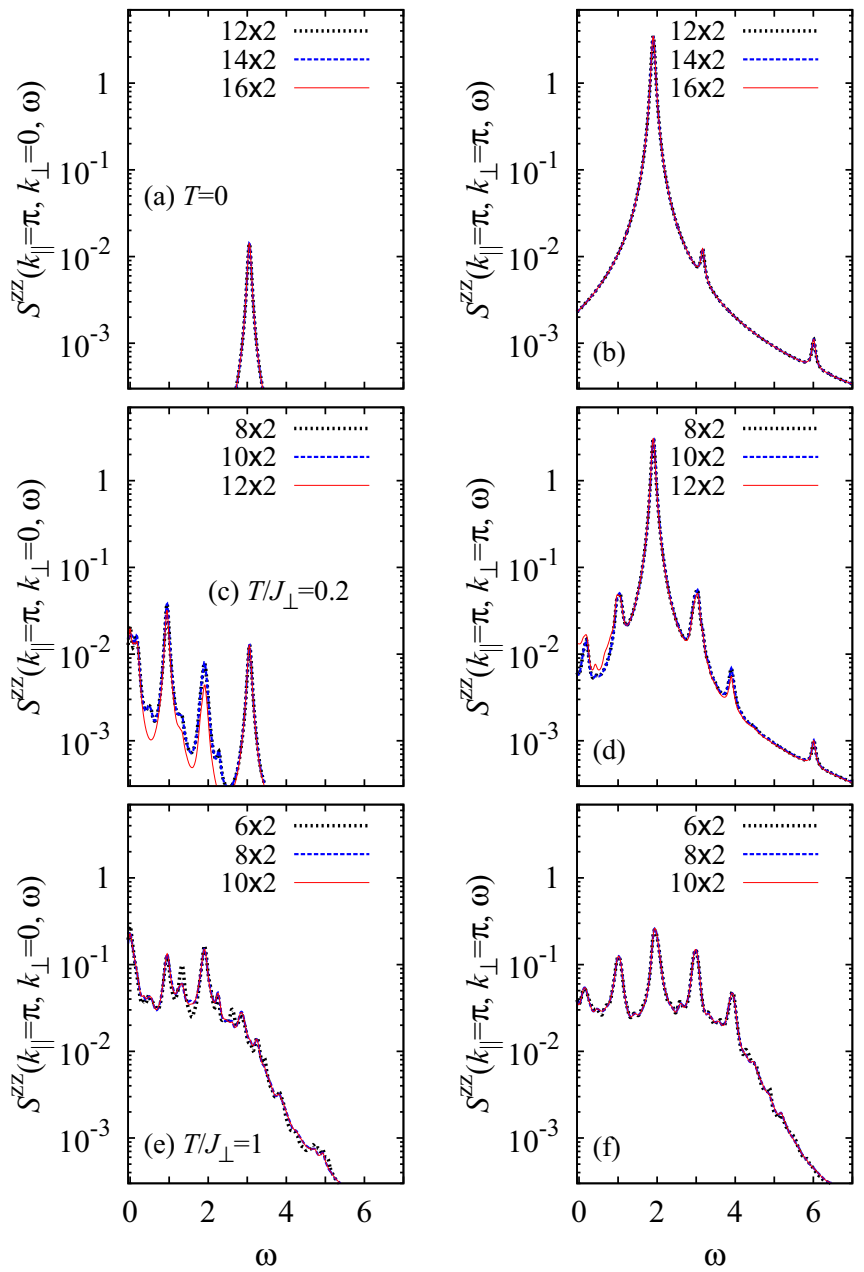

FIG. 13. Intensity at $k_{\|}=\pi$ in (a), (c), and (e) the symmetric and (b), (d), and (f) the antisymmetric channel, shown as a function of energy for the ladder of Fig. 1 with the system size specified, coupling parameters $J_{\perp}=2, J_{\|}=1$, and $J_{\times}=0.9$, and $\eta=J_{\|} / 20$, for temperatures of (a) and (b) $T / J_{\perp}=0$, (c) and (d) 0.2 , and (e) and (f) 1 .

we find the dominant excitations at $\omega / J_{\|}=0.55,0.45$, and 2.45 in Figs. 5 and 11.

The next higher energy levels, and presumably nextstrongest intensities, will arise from the $2 \rightarrow 3$ transitions, which are expected at the energies $\left|j^{\prime}-3\right|,\left|j^{\prime}-2\right|, j^{\prime}-1$, and so on. Many of these are not detectable because they contribute at exactly the same energies as the stronger $1 \rightarrow 2$ signal, and cannot be separated by using different values of $j^{\prime}$. The first nondegenerate option, the $j^{\prime}+2$ signal, is clearly visible in all of Figs. 3 (9), 5 (11), and 7 (13), the last with an inverted dispersion, but is definitely weaker than the $1 \rightarrow 2$ peaks at all temperatures. As noted above, processes involving four-triplet bound states appear at irrational energies and these form the leading contributions to the "background" of many discrete energies visible in all of the spectra shown in Figs. 3 to 5 and 7 even at moderate $T$.

The logical extension of this discussion is the obvious importance of "multiparticle" excitations, by which is meant the low-lying bound states of high- $n$ triplon clusters. Particularly

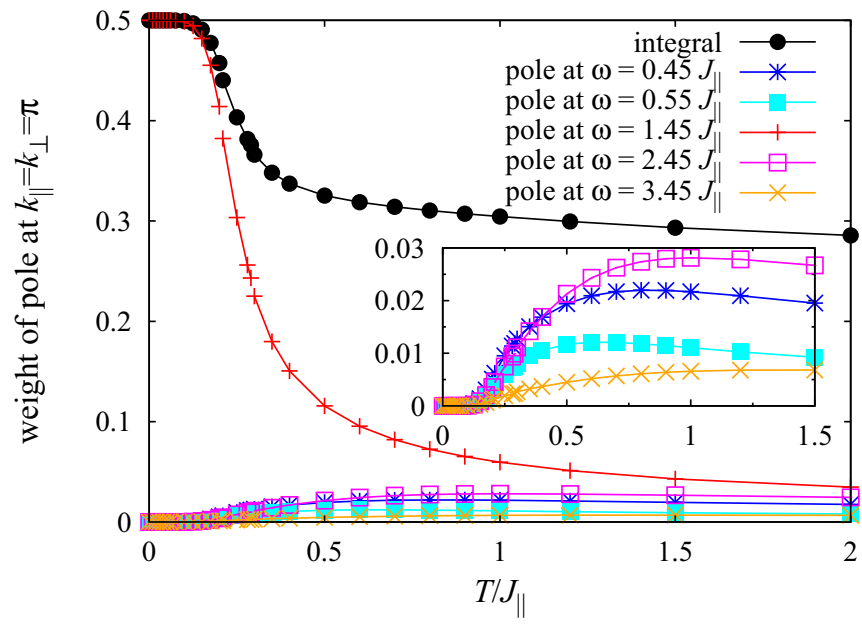

FIG. 14. Weights of selected poles in the dynamical structure factor in the antisymmetric channel for a fully frustrated ladder with $j^{\prime}=1.45$, shown as a function of temperature for the total spectral weight, the one-triplon mode, and the four most intense multi-triplon bound states. The thermal evolution of the bound-state intensities (inset) reflects the common origin of the three leading lines, which differs from that of the fourth (see text). System sizes are $L=14$ rungs at low $T$, followed by $L=12$ and then $L=10$ at higher temperatures; data for identical system sizes are connected by lines.

for values of $j^{\prime}$ just above $j_{c}^{\prime}$, as in Fig. 5, these cause the very rapid filling of the spectrum as the temperature is raised, with thermal excitations populating bound states over a wide range of $n$. These multi-triplon excitations are in fact rather spatially extended objects and as such constitute exceptions to the notion that all physics tends to be extremely local in highly frustrated and gapped systems. This is also the origin of problems with finite-size effects entering our calculations as $j^{\prime} \rightarrow j_{c}^{\prime}$, some of which are visible in Fig. 11.

\section{B. Spectral-weight redistribution}

The other primary aspect of our results that can be explained quantitatively is the redistribution of spectral weight among the different discrete excitations as a function of the increasing temperature. Concentrating again on the fully frustrated case and the antisymmetric channel, the weights of the poles for any wave vector in the spectral functions show (Figs. 9 and 11) a clear growth with temperature of the intensity in a number of satellite peaks at the expense of the one-triplon peak. This information is shown in Fig. 14 for the parameter choice $j^{\prime}=1.45$, where the three strongest satellites, at $\omega / J_{\|}=0.55$, 0.45 , and 2.45 , are respectively the transitions from the onetriplon band to the singlet, triplet, and quintet of the two-triplon bound state, and the fourth, at $\omega / J_{\|}=3.45$, contains the $j^{\prime}+2$ transitions within the set of $2 \rightarrow 3$ processes.

Appendix B analyzes the spectral weights of the $1 \rightarrow 2$ processes. Because all transitions are independent of the wave vector, the result is an extremely straightforward form where the bound-state intensities are related by simple ratios multiplying a thermal factor and a single matrix element. However, several features complicate efforts to apply this analysis to the observed spectral weights, shown in the inset 


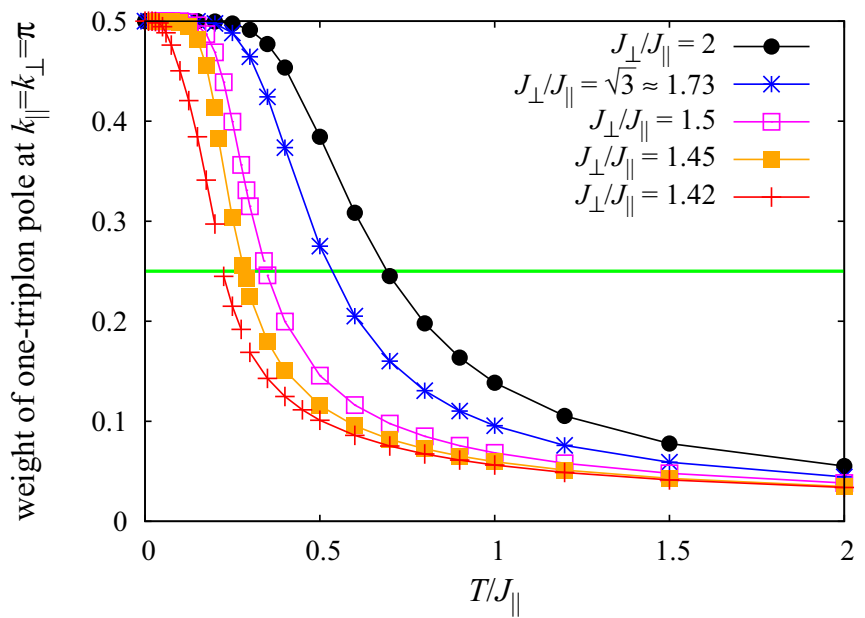

FIG. 15. Weight of the one-triplon pole in the dynamical structure factor of the fully frustrated ladder as a function of temperature for five different coupling ratios approaching the quantum critical point ( $\left.j_{c}^{\prime}=1.40148\right)$. The system sizes used in our calculations are $L=14$ at low temperatures for $j^{\prime}=1.42$ and $1.45, L=12$ at intermediate temperatures for $j^{\prime}=1.42$ and 1.45 and at low temperatures for $j^{\prime}=1.5$, and $L=10$ otherwise. The quantity $T_{1 / 2}$ is defined at a given coupling ratio, $j^{\prime}$, as the temperature where the intensity falls to half of its $T=0$ value (horizontal line).

of Fig. 14. One is the problem noted above, concerning the attribution of intensities due to the different, degenerate $1 \rightarrow 2$ and $2 \rightarrow 3$ processes. In principle, the intensity of each line $(i)$ should have a leading temperature dependence $I_{i}=a_{i} e^{-J_{\perp} / T}+b_{i} e^{-\left(2 J_{\perp}-l_{i} J_{\|}\right) / T}$, with related coefficients $a_{i}$ and $b_{i}$, and $l_{i}$ a small (positive, negative, or zero) integer. It is clear that the three leading lines in the inset of Fig. 14 cross each other at temperatures as low as $T=0.2 J_{\perp}$, and very obviously at $0.4 J_{\perp}$, as well as having maxima at quite different peak values. These results imply both strong effects from multiple contributions at rather low temperatures and additional physics at higher temperatures, where the satellites lose intensity again due to further thermal excitation. We draw attention also to the fact that the fourth (weakest) line in the inset, which from above is the strongest dominated by $2 \rightarrow 3$ processes, clearly has a thermal evolution characterized by an initial-state energy (presumably of order $2 J_{\perp}$ ) rather different from that $\left(J_{\perp}\right)$ governing the three $1 \rightarrow 2$ processes. Because thermal effects extend so rapidly beyond the lowest order, the relevance of an analysis of the type shown in Appendix B could be tested only at very low temperatures, perhaps around $0.1 J_{\perp}$. Finally, such a test could only be performed meaningfully at values of $j^{\prime}$ not too close to $j_{c}^{\prime}\left(j^{\prime}>1.45\right.$ for $L=14$ ladders), because otherwise the low-lying, Haldane-type intruder states mentioned in Sec. II also interfere with the overall intensities in our finite-size calculations.

\section{One-triplon spectral weight}

We turn next to the question motivating our entire investigation, namely the factors responsible for the anomalously rapid loss of spectral weight from the one-triplon band visible in a material such as $\mathrm{SrCu}_{2}\left(\mathrm{BO}_{3}\right)_{2}$. We focus again on the fully

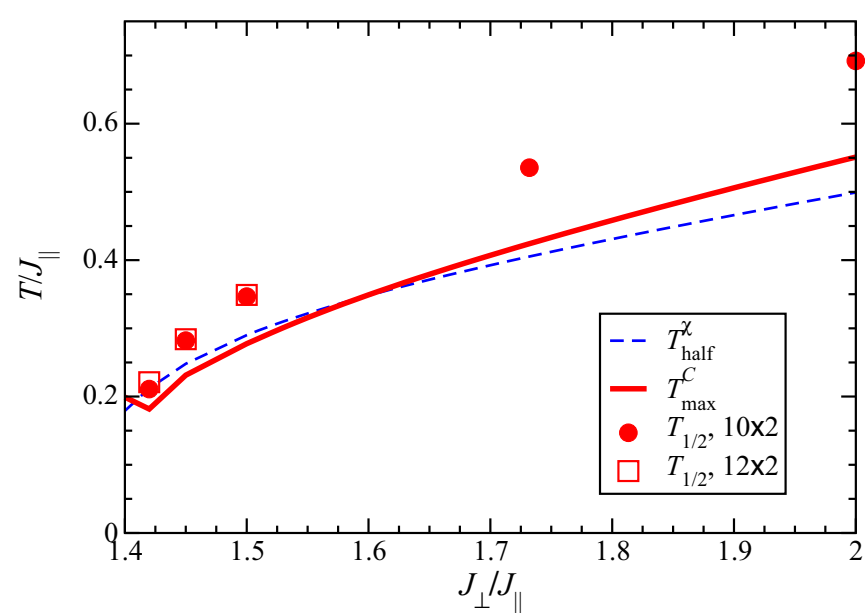

FIG. 16. Temperature $T_{1 / 2}\left(j^{\prime}\right)$, at which the weight of the dynamical structure factor (Fig. 15) falls to one half of its peak value, shown by the symbols for several values of the coupling ratio, $j^{\prime}$. Lines indicate the values of the quantities $T_{\max }^{C}$ and $T_{\text {half }}^{\chi}$ (see text) in the thermodynamic limit, deduced from the numerical results of Ref. [34] for the magnetic specific heat and susceptibility.

frustrated system. For all coupling ratios, $j^{\prime}$, there is a loss of weight from the one-triplon mode, which can be quantified from the weight of the one-triplon pole in Figs. 9 to 11. This is shown in Fig. 15 for several values of $j^{\prime}$; a discussion of finite-size effects in the context of this figure, specifically for the $j^{\prime}=1.42$ and 1.45 curves, is presented in Appendix C. The observed loss of intensity with temperature is not unusual, and its unconventional feature is only the way it happens, between discrete energy levels that remain sharp at all temperatures, rather than as a conventional thermal broadening (Figs. 6 and 12). Over much of the range of $j^{\prime}$, the temperature at which a significant loss of one-triplon spectral weight occurs cannot be said to be anomalously low, as shown in Fig. 15 for parameter values such as $j^{\prime}=2$. However, our single most important finding is that, close to $j_{c}^{\prime}$, there is a regime where large numbers of multiparticle excitations, involving many-rung triplon clusters in strongly bound and low-lying states (Fig. 2), come to dominate the low-energy physics [34]. As Fig. 15 shows clearly, these states do lead to rapid transfer of spectral weight out of the one-triplon band at anomalously low temperatures.

To quantify the loss of spectral weight from the one-triplon signal, we consider the temperature, $T_{1 / 2}$, at which the intensity falls to one half of its zero-temperature value, given by the intersection of the different curves with the horizontal line in Fig. 15. In Fig. 16, we show the values of $T_{1 / 2}$ as a function of $j^{\prime}$ in the rung-singlet regime, noting that the phase transition, $j_{c}^{\prime} \approx 1.4015$, lies essentially on the left boundary of the figure. It is important to note that, although finite-size effects are expected to be relevant for small values of $\left|j^{\prime}-j_{c}^{\prime}\right|$ (Sec. II, Appendix C), our results for $j^{\prime}=1.42,1.45$, and 1.5 from ladders of $L=12$, in addition to $L=10$, demonstrate that $T_{1 / 2}$ can still be extracted reliably in this regime with the available system sizes. At values of $j^{\prime}$ far from the transition, the one-triplon spectral weight remains close to unity until a temperature that is a significant fraction of $J_{\|}$, 
as can be seen by considering the case $j^{\prime}=2$ in Fig. 15, where $T_{1 / 2} \simeq 0.692 J_{\|}=0.346 J_{\perp}$ (Fig. 16). However, as $j^{\prime}$ approaches $j_{c}^{\prime}, T_{1 / 2}$ falls to an anomalously low temperature, of order $0.22 J_{\|} \approx 0.15 J_{\perp}$ when $j^{\prime}=1.42$.

As a means to gain some initial insight into this emerging low energy scale, in Fig. 16 we compare $T_{1 / 2}$ with the characteristic temperatures extracted from the thermodynamic response of the system. In Ref. [34], we showed that the magnetic specific heat $C(T)$ is characterized by a strong peak, which becomes narrower and moves to lower energies as $j^{\prime} \rightarrow j_{c}^{\prime}$, and in Fig. 16, we show its position, $T_{\max }^{C}$. The magnetic susceptibility $\chi(T)$ has a very broad peak, but can be characterized quite sensitively by its rapid onset, for which we use the temperature, $T_{\text {half }}^{\chi}$, where $\chi(T)$ reaches half of its peak height. Because $C(T)$ is a consequence of excitations to all available levels in the spectrum, whereas $\chi(T)$ is a consequence of excitations to all available magnetic levels $(S \geqslant 1)$, the fact that $T_{\max }^{C}$ and $T_{\text {half }}^{\chi}$ track each other very closely makes clear that the descent of singlet and triplet bound-state branches as $j^{\prime} \rightarrow j_{c}^{\prime}$ (Fig. 2) is very similar not only in energy but also in density. To lowest order, the temperature scale obtained from a dynamical quantity, $T_{1 / 2}$, is effectively identical in the vicinity of the transition to the characteristic temperatures obtained from the static response. This confirms that the plethora of low-lying bound states accessible at finite temperatures near the quantum phase transition is explicitly the origin of the rapid loss of onetriplon spectral weight. We comment here that a connection between $C(T)$ and rapid spectral-weight transfer, based on the presence of well-localized bound-state modes of the system, was mooted for $\mathrm{SrCu}_{2}\left(\mathrm{BO}_{3}\right)_{2}$ on the basis of Raman-scattering studies in Ref. [95]. That $T_{1 / 2}$ rises above $T_{\max }^{C}$ and $T_{\text {half }}^{\chi}$ in the regime far from $j_{c}^{\prime}$ (Fig. 16) suggests that the spectral-weight transfer depends more critically than do $C(T)$ and $\chi(T)$ on a smaller number of processes (i.e., on a low density of levels) whose gaps become large.

The origin of the emerging low energy scale around $j_{c}^{\prime}$ is somewhat complex, with two distinct types of contribution. In Ref. [34], we discussed the quantity $E_{\text {bond }}$, which is effectively the energy scale of a domain wall between the potentially long regions of singlet and triplet rungs that characterize the system in the vicinity of $j_{c}^{\prime}$. However, the peak in the specific heat $T_{\max }^{C}$ and the half-height temperature $T_{\text {half }}^{\chi}$ of the susceptibility are always irrational fractions of the characteristic energy scale, which remains on the order of the gap to the lowest excitations (Fig. 2). The second type of contribution is from the effective number of excited energy levels involved in draining weight away from the one-triplon branch, and this quantity remains difficult to define. A reasonable hypothesis for the rapid loss of spectral weight would be that pairs of triplons scatter strongly, with an effective scattering length significantly larger than the lattice constant [25], and the area of this scattering region could be used to deduce a number of states. Our analysis demonstrates that this is not the correct picture, in that the origin of the strong quasiparticle scattering lies in the presence of many multi-triplon bound states rather than in extended two-triplon ones. Although it is clear from the data of Fig. 15 that many states are involved in the loss of spectral weight, the deduction of an effective number of multi-triplon bound-state branches from these data remains an ill-defined task.
As to the destination of the spectral weight lost from the one-triplon branch, this weight is transferred systematically to the primary multi-triplon excitations, whose intensities grow exponentially at low temperatures (as discussed above and shown in the inset of Fig. 14) and then more slowly beyond $T / J_{\|} \approx 0.5$. Despite sometimes nonmonotonic behavior, these satellite peak intensities do approach a constant ratio at high temperatures, as we will show in Sec. IV D. If the ladder couplings are altered away from perfect frustration, this picture is altered due to the presence of some "continuum" thermal effects. However, it is difficult to find a meaningful comparison of spectral-weight shifts with the fully unfrustrated system, where there are no special mode energies; in this case it would be necessary to characterize the shift of intensity by integrating the spectrum over certain frequency windows, chosen on the basis of the low- $T$ spectra, giving another quite ill-defined procedure that would lose validity at higher temperatures.

In closing this section, we comment that Fig. 16 allows a qualitative analogy with the situation in $\mathrm{SrCu}_{2}\left(\mathrm{BO}_{3}\right)_{2}$. This material is known to be located close to a first-order quantum phase transition out of its rung-singlet phase (to a plaquette valence-bond state), and to have an anomalously low value, $T_{1 / 2} / \Delta_{0}=7 \mathrm{~K} / 35 \mathrm{~K}=0.2$ [25], of the ratio between the half-height temperature of the one-triplon intensity and the size of the one-triplon gap. The conventional discussion of this material is phrased using $1 / j^{\prime}$, with the phase transition occurring at $1 / j_{c}^{\prime}=0.675[96]$ and the best estimates of $1 / j^{\prime}$ $(=0.635[22,97])$ falling within $5 \%$ of this value. While it is tempting to place $\mathrm{SrCu}_{2}\left(\mathrm{BO}_{3}\right)_{2}$ directly on Fig. 16, where the ratio of 0.2 corresponds to $j^{\prime}=1.42$ and therefore lies very close to the transition, finding the limiting value of $T_{1 / 2} / \Delta_{0}$ for the Shastry-Sutherland system, analogous to that obtained here for the fully frustrated ladder, will require detailed calculations in a $2 \mathrm{D}$ model. In a very recent and exciting development [98], an applied pressure has been used to drive the $\mathrm{SrCu}_{2}\left(\mathrm{BO}_{3}\right)_{2}$ system through the quantum phase transition, i.e., to push $j^{\prime}$ across $j_{c}^{\prime}$, which raises the possibility of detailed thermodynamic and spectral-weight measurements as a function of the proximity, $\delta j^{\prime}=\left|j^{\prime}-j_{c}^{\prime}\right| / j_{c}^{\prime}$, to the transition.

\section{High-temperature spectra}

We conclude the analysis of our numerical results by discussing the situation at high temperatures. The results of Fig. 8 for the limit $T \rightarrow \infty$ are shown in Fig. 17 in the form of logarithmic intensities for the wave vector $k_{\|}=\pi$. For the fully frustrated ladders [Figs. 17(a)-17(f)], as noted in Sec. III, there are always special energies where the dynamical structure factor has a high spectral weight. This is a consequence of the discrete support, i.e., the $T=0$ spectrum of nondispersive bound-state energy levels arising from a hierarchy of $n$-triplon clusters, and of the matrix elements associated with transitions between these states [Appendix B]. For the unfrustrated ladder [Figs. 17(g) and 17(h)], this weight is spread out completely over the available states, which form continuous energy bands, but a plateau structure emerges in both the symmetric and antisymmetric channels. These plateaus may be ascribed to the scattering processes connecting sectors of different triplon number, $l$, specifically $0 \rightarrow 1,1 \rightarrow 2, \ldots$ in the antisymmetric channel and $0 \rightarrow 2,1 \rightarrow 3, \ldots$ in the symmetric channel. We 

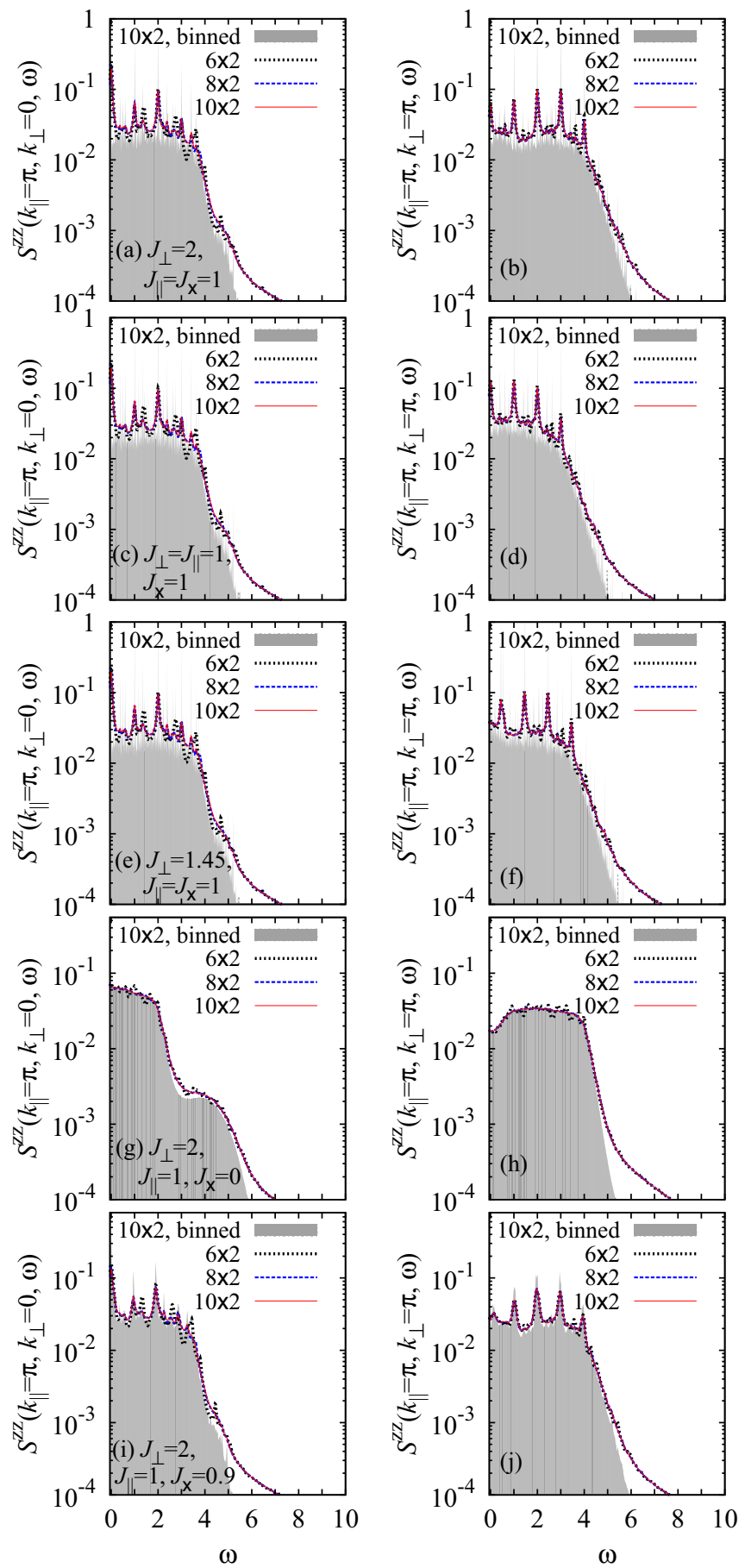

FIG. 17. Intensity at $k_{\|}=\pi$ in (a), (c), (e), (g), and (i) the symmetric and (b), (d), (f), (h), and (j) the antisymmetric channel, shown as a function of energy at infinite temperature for the ladder of Fig. 1 with coupling parameters $J_{\perp}=2$ and $J_{\|}=J_{\times}=1$ (a) and (b) $J_{\perp}=J_{\|}=J_{\times}=1$ (c) and (d); $J_{\perp}=1.45$ and $J_{\|}=J_{\times}=1$ (e) and (f); $J_{\perp}=2, J_{\|}=1$, and $J_{\times}=0(\mathrm{~g})$ and (h); $J_{\perp}=2, J_{\|}=1$, and $J_{\times}=0.9$ (i) and (j). The lines show ED data calculated for the specified system sizes with a Lorentzian broadening $\eta=J_{\|} / 20$; the grey shading shows binned data obtained from calculations on a ladder of $10 \times 2$ spins with $\eta=0$.

note that these processes are not symmetrical in energy, but that the reverse processes start to contribute at higher temperatures, and in fact dominate the low-energy response once many thermally occupied states are available to give up their energy.

Figure 17 may also be used to discuss the intrinsic line shape of the spectral features in spin ladders as a consequence of their frustration. To remove the effects of the Lorentzian broadening ( $\left.\eta=J_{\|} / 20\right)$ used in Figs. 3 to 13, we have summed the weights of all the poles, meaning the coefficients of all the $\delta$-functions obtained in Eq. (3) in the limit $\eta=0$, in bins of sizes $\Delta \omega / J_{\|}=0.006, \ldots, 0.015$ (depending on the specific ratio $j^{\prime}$ ) and we present the resulting histograms for ladders of $L=10$ rungs as the grey shaded regions in Fig. 17. Focusing on the antisymmetric channel $\left(k_{\perp}=\pi\right)$, we observe that in fully frustrated ladders $\left[J_{\times}=J_{\|}\right.$, Figs. $17(\mathrm{~b}), 17(\mathrm{~d})$, and $17(\mathrm{f})]$ the dominant modes of the dynamical spectral function (Sec. IV A) remain as $\delta$-functions up to $T=\infty$. This complete absence of thermal broadening is due to the complete flatness of the band, which is in one-to-one correspondence with the conservation of every rung spin, in the fully frustrated ladder. Figure 17(j) shows that a detuning of the frustration to $J_{\times}=0.9 J_{\|}$smooths the spectral function: the previously sharp lines are now subject to thermal broadening, which gives them an intrinsic line width, on the order of $0.1 J_{\|}$, in approximate agreement with the band width in this case. Considering finally the unfrustrated ladder $\left[J_{\times}=0\right.$, Fig. 17(h)], here the lines also disappear completely from the binned data, leaving only the same broad plateaus observed in the spectral function with Lorentzian broadening. We note also that the Lorentzian broadening gives rise to high-frequency tails in the spectral functions, which may be considered as artifacts by comparison with the much more rapid decay towards high frequencies found in the intrinsic line shape (again we remind the reader of the logarithmic scale on the intensity axes). Similar effects are also observed in the binned data at finite temperatures, $0<T<\infty$ (not shown); the examples shown in Fig. 17 for $T=\infty$ represent the upper limit on intrinsic thermal broadening.

\section{E. Experiment}

Finally, we close our discussion of the thermal redistribution of spectral weight in the dynamical structure factor of frustrated systems by considering the experimental situation. To the best of our knowledge, no material has yet been identified with the effective magnetic Hamiltonian of the fully frustrated spin ladder. However, many low-dimensional frustrated quantum magnets are known and many have very narrow excitation bands as a consequence of their frustration. Thus we expect our results to serve as a valuable paradigm for understanding the thermodynamic [34] and dynamical response of frustrated quantum spin systems.

Our primary motivation for this study was the anomalously rapid loss of spectral weight in the one-triplon excitation as a function of temperature, and we have identified the proximity to a quantum phase transition, with its accompanying plethora of multiparticle bound states, as a strong source of such behavior. However, it is not the only possible origin of anomalous effects on spectral weights, and a complete interpretation requires an account of other factors. The majority of low-dimensional quantum magnets have additional "anisotropic" terms in the magnetic Hamiltonian beyond the 
Heisenberg interaction. A good example is the spin-chain material $\mathrm{Cu}\left(\mathrm{C}_{6} \mathrm{D}_{5} \mathrm{COO}\right)_{2} \cdot 3 \mathrm{D}_{2} \mathrm{O}$ ( $\mathrm{Cu}$-benzoate), where the anomalous opening of a gap in an applied magnetic field [99] was explained by the presence of Dzyaloshinskii-Moriya (DM) interactions and $g$-tensor anisotropy [100,101]. Electron spin resonance (ESR) studies of $\mathrm{Cu}$-benzoate [102-104], $\mathrm{KCuGaF}_{6}$ [105], and $\mathrm{Cu}$-pyrimidine dinitrate $[66,106]$ report spectral weights that vanish rapidly with increasing temperature even in the absence of frustrating interactions, and these have been ascribed to the presence of breather and soliton modes in the effective quantum sine-Gordon model $[100,101]$ for the material. Although no comparison of the field-induced gap with the temperature has yet shown that the thermally induced loss of ESR spectral weight is truly anomalous, the presence of additional modes arising due to spin anisotropy in the Hamiltonian does serve as an additional possible route to rapid thermal decay. Similar thermal effects are thought to be present in the strong-leg ladder material $\left(\mathrm{C}_{7} \mathrm{H}_{10} \mathrm{~N}\right)_{2} \mathrm{CuBr}_{4}$ (DIMPY) [107].

The archetypal perfectly frustrated quantum magnet, whose dynamical response at finite temperature motivated this work, is the $2 \mathrm{D}$ material $\mathrm{SrCu}_{2}\left(\mathrm{BO}_{3}\right)_{2}$. This system has complete frustration in its Shastry-Sutherland geometry, a very flat one-triplon excitation, and also possesses significant DM interactions, ensuring a degree of anisotropy in spin space. Here we have shown that fully frustrated spin interactions have a pronounced effect in creating multi-triplon bound states, whose presence causes a significant damping and redistribution of the one-triplon spectral weight even at low temperatures. One of our primary findings is that this effect is dramatically stronger near the quantum phase transition out of the rung-singlet state, where many multiparticle excitations with anomalously low energies come to dominate the response of the system. It is known that $\mathrm{SrCu}_{2}\left(\mathrm{BO}_{3}\right)_{2}$ lies close to this transition in the Shastry-Sutherland model, which occurs at the ratio $1 / j^{\prime} \equiv J_{s} / J_{r} \simeq 0.675$ [96] of the square-lattice and rung couplings, and thus we strongly suspect that the emergence of many low-lying multi-triplon bound states is precisely the physics of this material. We point out that the measured decay rate of the one-triplon band intensity with temperature [24,25], compared with calculations for the Shastry-Sutherland model exactly analogous to Fig. 15, could be used to estimate very accurately the proximity, $\delta j^{\prime}$, of $\mathrm{SrCu}_{2}\left(\mathrm{BO}_{3}\right)_{2}$ to the critical point. Whether or not these generic properties of a fully frustrated, SU(2)-symmetric model are enhanced by DM interactions in causing the near-total destruction of the one-triplon mode at $T \approx \Delta / 3$ in $\mathrm{SrCu}_{2}\left(\mathrm{BO}_{3}\right)_{2}$ will require detailed analysis of anisotropic models.

\section{SUMMARY}

The dynamical response of low-dimensional quantum magnets presents a major challenge both to theoretical understanding and to the most advanced numerical methods. This is particularly true for highly frustrated spin systems, which are characterized by an almost flat one-triplon excitation band at zero temperature, where little insight is available either into the temperature dependence of this single-particle dispersion or into the multiparticle dynamics developing at finite temperature. We have investigated these questions using the example of the frustrated two-leg spin ladder and we present systematic numerical results from exact diagonalization for the dynamic structure factor as a function of temperature, coupling ratio, and degree of frustration.

We find that the fully frustrated system has a discrete spectrum of excitations. As the temperature is increased, spectral weight is transferred out of the one-triplon band to levels at a wide range of energies, including those below the one-particle gap; these are the energy levels of highly localized bound states involving clusters of multiple neighboring triplons. Close to the quantum phase transition at $j_{c}^{\prime}=1.4015$, the bound states lie very low in energy, they involve very large numbers of triplets, and the weight transfer is anomalously rapid. These many-particle excitations are extended objects and, as a consequence of the discrete spectrum, they persist as sharp spectral features even to infinite temperatures.

One may ask whether these two remarkable qualitative features, the rapid transfer of spectral weight at temperatures much lower than the one-triplon gap and the persistence of well-defined peaks at very high temperatures, are connected. In principle, the rapid transfer of spectral weight depends on having a large number of low-lying energy levels in the spectrum, whereas the well-defined peaks are a specific consequence of having completely flat (localized) bands. However, it remains an open question whether a truly high density of low-lying states could be achieved in a system with (weakly) dispersive bands or lacking an exact convergence of levels at a quantum phase transition, and the rate of spectral-weight transfer in such cases may be limited.

Nevertheless, the first of these remarkable features offers an explanation for the qualitative physics underlying the observation by inelastic neutron scattering studies of the lowdimensional frustrated material $\mathrm{SrCu}_{2}\left(\mathrm{BO}_{3}\right)_{2}$ that the intensity of the one-triplon band is completely dispersed to states at all energies even at temperatures only $1 / 3$ of its gap. The second of these features, namely the persistence of sharp excitation peaks at high temperatures in a fully frustrated system, remains to be investigated in experiment.

\section{ACKNOWLEDGMENTS}

We thank S. Wessel for helpful discussions. We are grateful to the HLRN Hannover for the allocation of CPU time. This work was supported by the Helmholtz Association via the Virtual Institute "New states of matter and their excitations," by the Swiss NSF, by the NSF of China under Grant 11174365 , and by the National Basic Research Program of the Chinese MoST under Grant 2012CB921704.

\section{APPENDIX A: MULTI-TRIPLON BOUND STATES}

The dynamical structure factor [Eq. (3)] is obtained from the action of local spin-raising and -lowering operators. Analytical insight into its nature for the fully frustrated ladder begins from the expression of the bound states in the basis of triplons (one-rung triplet excitations). As noted in Sec. II, in the case where the frustrating couplings $j^{\prime}=J_{\perp} / J_{\|}=J_{\perp} / J_{\times} \geqslant j_{c}^{\prime} \simeq$ 1.4015, two triplons on neighboring rungs form an exact bound state. This bound state consists of a singlet, which we denote $|2 s\rangle$, with energy $E_{2 s} / J_{\|}=2\left(j^{\prime}-1\right)$, a triplet, denoted 
$|2 t, m\rangle, m=-1,0,1$, with energy $E_{2 t} / J_{\|}=2 j^{\prime}-1$, and a quintet, $|2 q, m\rangle, m=-2,-1, \ldots, 2$, with $E_{2 q} / J_{\|}=2 j^{\prime}+1$. In the basis $\left|m_{i}, m_{i+1}\right\rangle, m_{i}=-1,0,1$, of two triplons on neighboring rungs, the nine components of the bound state are straightforward linear combinations of the nine two-triplon states. Omitting the rung indices and denoting $m=-1$ by $\overline{1}$, these are

$$
\begin{aligned}
|2 q, 2\rangle & =|11\rangle, \quad|2 q, 1\rangle=\frac{1}{\sqrt{2}}(|10\rangle+|01\rangle), \\
|2 q, 0\rangle & =\frac{1}{\sqrt{6}}(|1 \overline{1}\rangle+2|00\rangle+|\overline{1} 1\rangle), \\
|2 t, 1\rangle & =\frac{1}{\sqrt{2}}(|10\rangle-|01\rangle), \\
|2 t, 0\rangle & =\frac{1}{\sqrt{2}}(|1 \overline{1}\rangle-|\overline{1} 1\rangle), \\
|2 s\rangle & =\frac{1}{\sqrt{3}}(|1 \overline{1}\rangle-|00\rangle+|\overline{1} 1\rangle),
\end{aligned}
$$

and symmetrically for the states $|2 q,-1\rangle,|2 q,-2\rangle$, and $|2 t,-1\rangle$.

In the same way, the 27 components of states with three triplons on neighboring rungs also form a set of multiplets, or effective three-triplon bound states. Diagonalizing the three-rung Hamiltonian in the different $S_{z}$ sectors yields the energies $E_{3 h} / J_{\|}=3 j^{\prime}+2$ (heptet, $|3 h, m\rangle$ ), $E_{3 q a} / J_{\|}=$ $3 j^{\prime}+1$ (quintet, $|3 q a, m\rangle$ ), $E_{3 t a} / J_{\|}=3 j^{\prime}$ (triplet, $|3 t a, m\rangle$ ), $E_{3 q b} / J_{\|}=E_{3 t b} / J_{\|}=3 j^{\prime}-1$ (degenerate quintet, $|3 q b, m\rangle$, and triplet, $|3 t b, m\rangle), E_{3 s} / J_{\|}=3 j^{\prime}-2$ (singlet, $|3 s\rangle$ ), and $E_{3 t c} / J_{\|}=3 j^{\prime}-3$ (triplet, $|3 t c, m\rangle$ ). We draw attention to the fact that the lowest-lying state of the multiplet is a triplet, and that this is a generic property of all odd-length multi-triplon clusters $[34,65]$. The wave functions of these states may be expressed as

$$
\begin{aligned}
|3 h, 3\rangle= & |111\rangle, \\
|3 h, 2\rangle= & \frac{1}{\sqrt{3}}(|110\rangle+|101\rangle+|011\rangle), \\
|3 h, 1\rangle= & \frac{1}{\sqrt{15}}(2|100\rangle+2|010\rangle+2|001\rangle \\
& +|11 \overline{1}\rangle+|1 \overline{1} 1\rangle+|\overline{1} 11\rangle), \\
|3 h, 0\rangle= & \frac{1}{\sqrt{10}}(|10 \overline{1}\rangle+|0 \overline{1} 1\rangle+|\overline{1} 10\rangle \\
& +2|000\rangle+|1 \overline{1} 0\rangle+|01 \overline{1}\rangle+|\overline{1} 01\rangle),
\end{aligned}
$$

for the heptet, with symmetrical expressions for $m=-1,-2$, and -3 ,

$$
\begin{aligned}
|3 q a, 2\rangle= & \frac{1}{\sqrt{2}}(|110\rangle-|011\rangle), \\
|3 q a, 1\rangle= & \frac{1}{2}(|100\rangle+|11 \overline{1}\rangle-|\overline{1} 11\rangle-|001\rangle), \\
|3 q a, 0\rangle= & \frac{1}{2 \sqrt{3}}(2|10 \overline{1}\rangle-|0 \overline{1} 1\rangle-|\overline{1} 10\rangle \\
& +|1 \overline{1} 0\rangle+|01 \overline{1}\rangle-2|\overline{1} 01\rangle), \\
|3 q b, 2\rangle= & \frac{1}{\sqrt{6}}(|110\rangle-2|101\rangle+|011\rangle), \\
|3 q b, 1\rangle= & \frac{1}{2 \sqrt{3}}(|100\rangle-2|010\rangle+|001\rangle \\
& -|11 \overline{1}\rangle+2|1 \overline{1} 1\rangle-|\overline{1} 11\rangle), \\
|3 q b, 0\rangle= & \frac{1}{2}(|1 \overline{1} 0\rangle-|01 \overline{1}\rangle-|\overline{1} 10\rangle+|0 \overline{1} 1\rangle),
\end{aligned}
$$

for the quintets,

$$
\begin{aligned}
|3 t a, 1\rangle= & \frac{1}{\sqrt{3}}(|010\rangle-|11 \overline{1}\rangle-|\overline{1} 11\rangle), \\
|3 t a, 0\rangle= & \frac{1}{\sqrt{3}}(|000\rangle-|10 \overline{1}\rangle-|\overline{1} 01\rangle), \\
|3 t b, 1\rangle= & \frac{1}{2}(|100\rangle-|001\rangle-|11 \overline{1}\rangle+|\overline{1} 11\rangle), \\
|3 t b, 0\rangle= & \frac{1}{2}(|1 \overline{1} 0\rangle-|01 \overline{1}\rangle-|0 \overline{1} 1\rangle+|\overline{1} 10\rangle), \\
|3 t c, 1\rangle= & \frac{1}{2 \sqrt{15}}(-3|100\rangle+2|010\rangle-3|001\rangle \\
& +|11 \overline{1}\rangle+6|1 \overline{1} 1\rangle+|\overline{1} 11\rangle), \\
|3 t c, 0\rangle= & \frac{1}{2 \sqrt{15}}(-2|10 \overline{1}\rangle+3|0 \overline{1} 1\rangle+3|\overline{1} 10\rangle \\
& -4|000\rangle+3|1 \overline{1} 0\rangle+3|01 \overline{1}\rangle-2|\overline{1} 01\rangle),
\end{aligned}
$$

for the triplets, and

$$
\begin{aligned}
|3 s\rangle= & \frac{1}{\sqrt{6}}(|10 \overline{1}\rangle+|0 \overline{1} 1\rangle+|\overline{1} 10\rangle \\
& -|1 \overline{1} 0\rangle-|01 \overline{1}\rangle-|\overline{1} 01\rangle)
\end{aligned}
$$

for the singlet.

One may continue the process for all higher- $n$ multiplets. Results for $n=4$ are obtained most readily by numerical diagonalization of the four-site Haldane chain with open boundary conditions (Sec. II) [34]. We do not find strong contributions from four-triplon bound states in the dynamical response and do not enter into further detail here. We note only that the two lowest-lying states of the multiplet are a total singlet with energy $E_{4 s}^{1} / J_{\|}=4 j^{\prime}-4.64575$ and a triplet with $E_{4 t}^{1} / J_{\|}=4 j^{\prime}-4.13658$, with the singlet the lowest level as for all even- $n$ bound states.

\section{APPENDIX B: DYNAMICAL SPECTRAL FUNCTION}

We consider the analytical calculation of the contributions from small- $n$ bound states to the dynamical spectral function in the antisymmetric channel. In its most general form,

$S^{\alpha \beta}(\mathbf{q}, \omega, T)=\sum_{i j} p_{i}\left\langle i\left|S_{\mathbf{q}}^{\alpha}\right| j\right\rangle\left\langle j\left|S_{\mathbf{q}}^{\beta}\right| i\right\rangle \delta\left(\omega-E_{j}+E_{i}\right)$,

where $|i\rangle$ and $|j\rangle$ denote the initial and final states of the scattering process. For a system with a Heisenberg Hamiltonian [preserving SU(2) symmetry], $S^{\alpha \beta}(\mathbf{q}, \omega, T)$ obeys the symmetries $S^{x x}=S^{y y}=S^{z z}=S^{+-}=S^{-+}$, and here it is most straightforward to obtain $S^{z z}$ (3) from $S^{+-}$. The form of Eq. (3) is obtained by considering the delta function as $\pi \delta(E)=\lim _{\eta \rightarrow 0} \operatorname{Im}[E-i \eta]^{-1}$. The temperature dependence of the dynamical spectral function is contained within the energy $\delta$-function and in the probability function $p_{i}=e^{-E_{i} / T} / Z(T)$ for the occupation of the initial state.

In this Appendix, we specialize to the case of the dynamical structure factor for inelastic neutron scattering. In the highly restricted single-rung basis, a neutron spin-flip changes both the $S$ and $S_{z}$ quantum numbers by \pm 1 , also changing the rung parity, and therefore the corresponding $S^{\alpha \beta}$ appears exclusively in the antisymmetric channel (Secs. III and IV). An incident neutron may either alter the triplon number, explicitly changing the state of the ladder between two different sectors with $l$ and $l+1$ triplons, or may cause excitations within single 
bound-state multiplets of $n \geqslant 2$. Because the latter require higher activation energies $\left[E_{i}\right.$ in Eq. (B1)] than processes with zero or one initial triplons, they are significantly weaker and will not be considered explicitly here. As noted above, we also restrict our presentation to the fully frustrated ladder, where the fact that the band energies are completely independent of the wave vector, $k_{\|}$, means that the real-space matrix elements provide the full information required. Thus we may consider only the action of a neutron incident on a single ladder rung, whose scattering matrix element from the zero- to the onetriplon sector $(0 \rightarrow 1)$ is given by $M=\left\langle 1\left|S^{+}\right| 0\right\rangle$, to deduce the relative intensities of all higher processes $(l \rightarrow l+1)$.

The calculation of $S^{+-}(\mathbf{q}, \omega, T)$ for these cases proceeds directly from the wave functions specified in Eqs. (A1)-(A8). The case $1 \rightarrow 2$ involves scattering of a neutron incident on a singlet rung, one of whose neighbors is a triplon. The final state of these two ladder rungs is then one of the bound states of two triplons specified in Eq. (A1). The probability of finding a suitable initial state with an isolated triplon on site $i+1$ is given by

$$
2 p_{i}(T)=2 p_{t}(T) p_{s}(T)=2 e^{-J_{\perp} / T} / Z,
$$

where $p_{t}=e^{-J_{\perp} / T}$ is the probability for a rung triplon excitation, $p_{s} \equiv 1 / Z$ is the (temperature-dependent) probability of the neighboring rung to be in its singlet (i.e., unexcited) state, and the factor of 2 is for the two possible processes of exciting the neighboring rung singlet.

In the basis of rung quantum numbers, a singlet excited to the triplet state with $m_{i}=1$, and with a triplet of arbitrary $m_{i+1}$ located at site $i+1$, results in the two-rung states $|1,1\rangle$, $|1,0\rangle$, and $|1, \overline{1}\rangle$. Clearly, the first is identically the two-rung state $|2 q, 2\rangle$, the second may be part of the state $|2 q, 1\rangle$ or $|2 t, 1\rangle$ [each, from Eq. (A1), with probability $1 / 2$ ], and the third may be part of the states $|2 q, 0\rangle,|2 t, 0\rangle$, or $|2 s, 0\rangle$ (with respective probabilities $1 / 6,1 / 2$, and $1 / 3$ ). In the basis of multiplet quantum numbers, one finds the conventional, less restrictive selection rules for neutron scattering, $\Delta S=\Delta S_{z}=0$, \pm 1 . The contributions of one- to two-triplon scattering processes to the dynamical structure factor are then

$$
\begin{aligned}
& S_{|1 t\rangle \rightarrow|2 q, 2\rangle}^{+-}(q, \omega, T)=2 p_{t}(T) p_{s}(T)|M|^{2} \delta\left(\omega-J-J^{\prime}\right), \\
& S_{|1 t\rangle \rightarrow|2 q, 1\rangle}^{+-}(q, \omega, T)=p_{t}(T) p_{s}(T)|M|^{2} \delta\left(\omega-J-J^{\prime}\right), \\
& S_{|1 t\rangle \rightarrow|2 q, 0\rangle}^{+-}(q, \omega, T)=p_{t}(T) p_{s}(T)|M|^{2} \delta\left(\omega-J-J^{\prime}\right), \\
& S_{|1 t\rangle \rightarrow|2 t, 1\rangle}^{+-}(q, \omega, T)=\frac{1}{3} p_{t}(T) p_{s}(T)|M|^{2} \delta\left(\omega-J+J^{\prime}\right), \\
& S_{|1 t\rangle \rightarrow|2 t, 0\rangle}^{+-}(q, \omega, T)=p_{t}(T) p_{s}(T)|M|^{2} \delta\left(\omega-J+J^{\prime}\right), \\
& S_{|1 t\rangle \rightarrow|2 s, 0\rangle}^{+-}(q, \omega, T)=\frac{2}{3} p_{t}(T) p_{s}(T)|M|^{2} \delta\left(\omega-J+2 J^{\prime}\right),
\end{aligned}
$$

all independent of the neutron wave vector, $q$. We note in addition that these expressions contain, as specified in Eq. (B1), no explicit dependence of the intensity on the final-state energy, and thus all the branches of each $n$-triplon bound state have similar probabilities in the same sector (differing only by the coefficients in their wave functions). We draw attention to the fact that, although the scattering energy, $\omega$, in Eqs. (B3) can become negative for $|1 t\rangle \rightarrow|2 s, 0\rangle$ processes in the range $j_{c}^{\prime}<j^{\prime}<2$, the excitation energy for the initial state remains positive; this is indeed the dominant process observed in Secs. III and IV to gain spectral weight at finite temperatures.

A similar exercise can be applied to deduce the probabilities of the two-triplon bound states and, with an additional excited triplet, their overlap with the three-triplon bound states [Eqs. (A2)-(A8)], to obtain the complete contribution to the dynamical structure factor from $2 \rightarrow 3$ processes. Although some of these are visible in our results at intermediate temperatures, as discussed in Sec. IV, their contributions remain small compared to $1 \rightarrow 2$ processes. Thus their energies are visible but their intensities are difficult to characterize quantitatively, and so we do not perform the spectral-weight calculation explicitly here.

\section{APPENDIX C: FINITE-SIZE ANALYSIS OF THE ONE-TRIPLON SPECTRAL WEIGHT}

We consider the finite-size effects in our calculation of $S^{z z}\left(k_{\|}=\pi, k_{\perp}=\pi, \omega\right)$ close to the critical point, $j_{c}^{\prime}$, of the fully frustrated ladder $\left(J_{\times}=J_{\|}\right)$. Among all of the features studied, the spectral weight of the one-triplon excitation, appearing at $\omega=J_{\perp}$, exhibits the most severe finite-size effects, and so we focus on this quantity. At $T=0$, the pole at $\omega=J_{\perp}$ in $S^{z z}\left(k_{\|}, k_{\perp}=\pi, \omega\right)$ has a weight of $1 / 2$, irrespective of the ladder length, whence finite-size effects appear only for $T>0$. Also at high $T$, any dependence of our results on the system size is weak and as a consequence we may restrict our focus further to the region of low but finite temperatures.

The primary problem in the calculation of the spectral weight is caused by the presence of the intruder states discussed in Sec. II and illustrated in Fig. 2. For coupling ratios $j^{\prime}=1.45$ and 1.42 , the spurious Haldane ground state lies below the first true excitation of the rung-singlet phase for ladders with $L \leqslant 14$ rungs, as shown by the symbols in Fig. 2. Nevertheless, this state is nondegenerate, whereas the true low-lying excitations are at least $L$-fold degenerate, and thus we will find that systems with $L \leqslant 14$ rungs are in fact sufficient to approximate the thermodynamic limit.

Figure 18 shows the case $j^{\prime}=1.45$. As a gauge of the temperatures at which finite-size effects matter, the $L=6$ ladder can be considered as converged to the thermodynamic limit for $T / J_{\|} \gtrsim 0.5$ and the $L=10$ ladder as a good approximation to the infinite system for $T / J_{\|} \gtrsim 0.35$. At lower temperatures, our data for ladders of $L=12$ and 14 rungs show that finite-size effects remain visible, but that corrections between $L=10$ and 14 are small (of order 5\%) and converging. Overall, the largest available system presents a reasonable approximation to the thermodynamic limit for each temperature shown in Fig. 18.

Figure 19 shows the case $j^{\prime}=1.42$, where the coupling ratio approaches the critical point. Because the finite-size critical value for the $L=6$ ladder is $j_{c}^{\prime}=1.436, j^{\prime}=1.42$ lies on the rung-triplet side of the transition for this system and the low-energy spectrum is completely different from the rung-singlet side; the $L=6$ data must therefore be excluded from the analysis of $j^{\prime}=1.42$. In this case, the $L=10$ ladder remains a good approximation to the infinite system for $T / J_{\|} \gtrsim 0.3$. The situation at temperatures below $T / J_{\|} \lesssim 0.25$ cannot be said to approach convergence for 


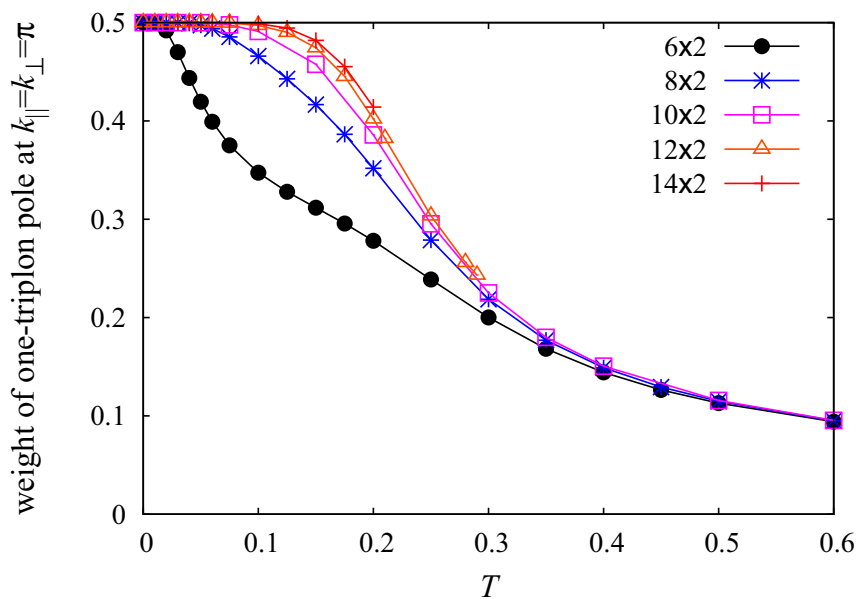

FIG. 18. Dependence on temperature of the weight of the onetriplon pole in the dynamical structure factor calculated for fully frustrated ladders of sizes $6 \leqslant L \leqslant 14$ rungs and with couplings $J_{\perp}=1.45$ and $J_{\|}=J_{\times}=1$.

ladders up to $L=14$. If one were to analyze the onset of the drop in spectral weight, which the $L=14$ ladder places in the region $0.02 \lesssim T / J_{\|} \lesssim 0.04$, this would in all probability underestimate the temperature scale that would be extracted

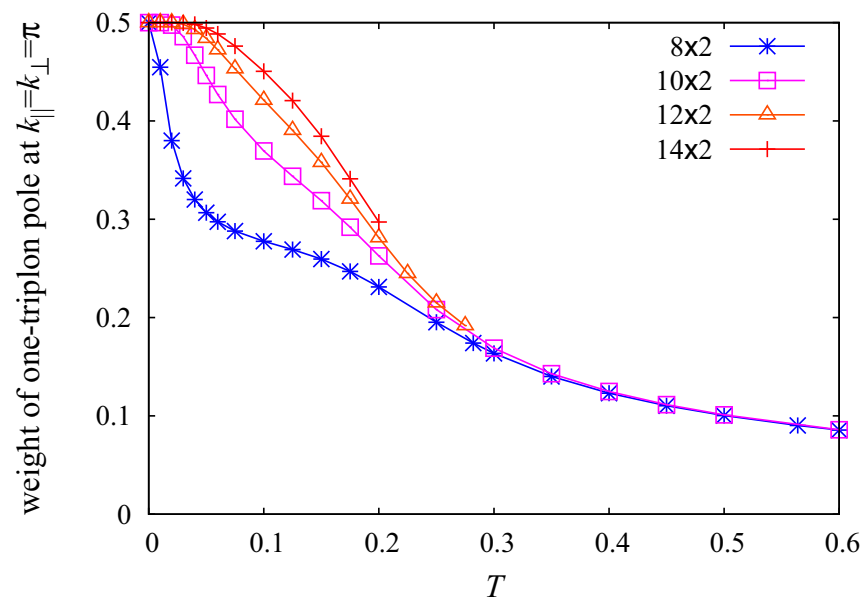

FIG. 19. Dependence on temperature of the weight of the onetriplon pole in the dynamical structure factor calculated for fully frustrated ladders of sizes $8 \leqslant L \leqslant 14$ rungs and with couplings $J_{\perp}=1.42$ and $J_{\|}=J_{\times}=1$.

from studies of longer ladders. However, for the temperature scale $T_{1 / 2}$ discussed in the main text, both the 12 - and even 10-rung ladders appear to provide a satisfactory approximation to the thermodynamic limit, $T_{1 / 2} \approx 0.225 J_{\|}$.
[1] H.-J. Mikeska and A. K. Kolezhuk, Lect. Notes Phys. 645, 1 (2004).

[2] L. D. Faddeev and L. A. Takhtajan, Phys. Lett. A 85, 375 (1981).

[3] E. Dagotto, J. Riera, and D. Scalapino, Phys. Rev. B 45, 5744 (1992).

[4] E. Dagotto and T. M. Rice, Science 271, 618 (1996).

[5] E. Dagotto, Rep. Prog. Phys. 62, 1525 (1999).

[6] F. D. M. Haldane, Phys. Rev. Lett. 50, 1153 (1983); Phys. Lett. A 93, 464 (1983).

[7] C. K. Majumdar and D. K. Ghosh, J. Math. Phys. 10, 1388 (1969).

[8] C. K. Majumdar, J. Phys. C: Solid State Phys. 3, 911 (1969).

[9] B. S. Shastry and B. Sutherland, Phys. Rev. Lett. 47, 964 (1981).

[10] C. Broholm and G. Aeppli, in Strong Interactions in Low Dimensions, edited by D. Baeriswyl and L. Degeorgi (Kluwer, Dordrecht, 2005).

[11] B. Normand and Ch. Rüegg, Phys. Rev. B 83, 054415 (2011).

[12] Ch. Rüegg, B. Normand, M. Matsumoto, Ch. Niedermayer, A. Furrer, K. W. Krämer, H.-U. Güdel, Ph. Bourges, Y. Sidis, and H. Mutka, Phys. Rev. Lett. 95, 267201 (2005).

[13] H. J. Mikeska and C. Luckmann, Phys. Rev. B 73, 184426 (2006).

[14] F. H. L. Essler and R. M. Konik, Phys. Rev. B 78, 100403(R) (2008); A. J. A. James, F. H. L. Essler, and R. M. Konik, ibid. 78, 094411 (2008); W. D. Goetze, U. Karahasanovic, and F. H. L. Essler, ibid. 82, 104417 (2010).

[15] A. James, Ph.D. thesis, University of Oxford, 2008.
[16] B. Fauseweh, J. Stolze, and G. S. Uhrig, Phys. Rev. B 90, 024428 (2014).

[17] B. Fauseweh and G. S. Uhrig, Phys. Rev. B 92, 214417 (2015).

[18] D. A. Tennant, B. Lake, A. J. A. James, F. H. L. Essler, S. Notbohm, H.-J. Mikeska, J. Fielden, P. Kögerler, P. C. Canfield, and M. T. F. Telling, Phys. Rev. B 85, 014402 (2012).

[19] E. S. Klyushina, A. C. Tiegel, B. Fauseweh, A. T. M. N. Islam, J. T. Park, B. Klemke, A. Honecker, G. S. Uhrig, S. R. Manmana, and B. Lake, Phys. Rev. B 93, 241109(R) (2016).

[20] B. S. Shastry and B. Sutherland, Physica B \& C 108, 1069 (1981)

[21] C. Knetter, A. Bühler, E. Müller-Hartmann, and G. S. Uhrig, Phys. Rev. Lett. 85, 3958 (2000).

[22] S. Miyahara and K. Ueda, J. Phys.: Condens. Matter 15, R327 (2003).

[23] H. Kageyama, M. Nishi, N. Aso, K. Onizuka, T. Yosihama, K. Nukui, K. Kodama, K. Kakurai, and Y. Ueda, Phys. Rev. Lett. 84, 5876 (2000).

[24] B. D. Gaulin, S. H. Lee, S. Haravifard, J. P. Castellan, A. J. Berlinsky, H. A. Dabkowska, Y. Qiu, and J. R. D. Copley, Phys. Rev. Lett. 93, 267202 (2004).

[25] M. E. Zayed, Ch. Rüegg, Th. Strässle, U. Stuhr, B. Roessli, M. Ay, J. Mesot, P. Link, E. Pomjakushina, M. Stingaciu, K. Conder, and H. M. Rønnow, Phys. Rev. Lett. 113, 067201 (2014).

[26] S. El Shawish, J. Bonča, and I. Sega, Phys. Rev. B 72, 184409 (2005)

[27] M. Takahashi, Thermodynamics of One-Dimensional Solvable Models (Cambridge University Press, Cambridge, 1999).

[28] M. Gaudin, Phys. Rev. Lett. 26, 1301 (1971). 
[29] S. Eggert, I. Affleck, and M. Takahashi, Phys. Rev. Lett. 73, 332 (1994).

[30] A. Klümper, Eur. Phys. J. B 5, 677 (1998).

[31] A. Klümper and D. C. Johnston, Phys. Rev. Lett. 84, 4701 (2000).

[32] C. Trippe, A. Honecker, A. Klümper, and V. Ohanyan, Phys. Rev. B 81, 054402 (2010).

[33] J. C. Bonner and M. E. Fisher, Phys. Rev. 135, A640 (1964).

[34] A. Honecker, S. Wessel, R. Kerkdyk, T. Pruschke, F. Mila, and B. Normand, Phys. Rev. B 93, 054408 (2016).

[35] F. Heidrich-Meisner, A. Honecker, and T. Vekua, Phys. Rev. B 74, 020403(R) (2006).

[36] A. Niazi, S. L. Bud'ko, D. L. Schlagel, J. Q. Yan, T. A. Lograsso, A. Kreyssig, S. Das, S. Nandi, A. I. Goldman, A. Honecker, R. W. McCallum, M. Reehuis, O. Pieper, B. Lake, and D. C. Johnston, Phys. Rev. B 79, 104432 (2009).

[37] B. Frischmuth, B. Ammon, and M. Troyer, Phys. Rev. B 54, R3714 (1996).

[38] D. C. Johnston, M. Troyer, S. Miyahara, D. Lidsky, K. Ueda, M. Azuma, Z. Hiroi, M. Takano, M. Isobe, Y. Ueda, M. A. Korotin, V. I. Anisimov, A. V. Mahajan, and L. L. Miller, arXiv:cond-mat/0001147v1.

[39] T. Nakamura, Phys. Rev. B 57, R3197 (1998).

[40] F. Alet, K. Damle, and S. Pujari, arXiv:1511.01586v1.

[41] X. Wang and T. Xiang, Phys. Rev. B 56, 5061 (1997).

[42] T. Xiang, Phys. Rev. B 58, 9142 (1998).

[43] K. Maisinger and U. Schollwöck, Phys. Rev. Lett. 81, 445 (1998).

[44] H. T. Lu, Y. J. Wang, S. Qin, and T. Xiang, Phys. Rev. B 74, 134425 (2006).

[45] S. Takemura and Y. Fukumoto, arXiv:cond-mat/0703260.

[46] J. Sirker, Phys. Rev. B 81, 014419 (2010).

[47] M. P. Gelfand, Phys. Rev. B 43, 8644 (1991).

[48] Y. Xian, Phys. Rev. B 52, 12485 (1995).

[49] A. Honecker, F. Mila, and M. Troyer, Eur. Phys. J. B 15, 227 (2000).

[50] X.-H. Chen, S. Y. Cho, H.-Q. Zhou, and M. T. Batchelor, J. Korean Phys. Soc. 68, 1114 (2016).

[51] S. R. White and D. A. Huse, Phys. Rev. B 48, 3844 (1993).

[52] O. Golinelli, Th. Jolicoeur, and R. Lacaze, Phys. Rev. B 50, 3037 (1994).

[53] I. Bose and S. Gayen, Phys. Rev. B 48, 10653(R) (1993).

[54] Zheng Weihong, V. Kotov, and J. Oitmaa, Phys. Rev. B 57, 11439 (1998).

[55] V. N. Kotov, O. P. Sushkov, and R. Eder, Phys. Rev. B 59, 6266 (1999).

[56] X. Wang, Mod. Phys. Lett. B 14, 327 (2000).

[57] W. Brenig and K. W. Becker, Phys. Rev. B 64, 214413 (2001).

[58] K. Totsuka and H.-J. Mikeska, Phys. Rev. B 66, 054435 (2002).

[59] T. Vekua and A. Honecker, Phys. Rev. B 73, 214427 (2006).

[60] E. H. Kim, Ö. Legeza, and J. Sólyom, Phys. Rev. B 77, 205121 (2008).

[61] T. Hikihara and O. A. Starykh, Phys. Rev. B 81, 064432 (2010).

[62] D. Poilblanc, Phys. Rev. Lett. 105, 077202 (2010).

[63] C. A. Lamas and J. M. Matera, Phys. Rev. B 92, 115111 (2015).

[64] T. Kennedy, J. Phys.: Condens. Matter 2, 5737 (1990).

[65] H. Niggemann, G. Uimin, and J. Zittartz, J. Phys.: Condens. Matter 9, 9031 (1997).
[66] A. C. Tiegel, A. Honecker, T. Pruschke, A. Ponomaryov, S. A. Zvyagin, R. Feyerherm, and S. R. Manmana, Phys. Rev. B 93, 104411 (2016).

[67] S. R. White, Phys. Rev. Lett. 69, 2863 (1992).

[68] S. R. White, Phys. Rev. B 48, 10345 (1993).

[69] U. Schollwöck, Ann. Phys. (NY) 326, 96 (2011).

[70] F. Naef, X. Wang, X. Zotos, and W. von der Linden, Phys. Rev. B 60, 359 (1999).

[71] J. Sirker and A. Klümper, Phys. Rev. B 71, 241101(R) (2005).

[72] M. Binder and T. Barthel, Phys. Rev. B 92, 125119 (2015).

[73] B. Bruognolo, J. von Delft, and A. Weichselbaum, Phys. Rev. B 92, 115105 (2015).

[74] F. Verstraete, J. J. García-Ripoll, and J. I. Cirac, Phys. Rev. Lett. 93, 207204 (2004).

[75] M. Zwolak and G. Vidal, Phys. Rev. Lett. 93, 207205 (2004).

[76] A. E. Feiguin and S. R. White, Phys. Rev. B 72, 220401(R) (2005).

[77] T. Barthel, U. Schollwöck, and S. R. White, Phys. Rev. B 79, 245101 (2009).

[78] C. Karrasch, J. H. Bardarson, and J. E. Moore, Phys. Rev. Lett. 108, 227206 (2012).

[79] T. Barthel, New J. Phys. 15, 073010 (2013).

[80] C. Karrasch, J. H. Bardarson, and J. E. Moore, New J. Phys. 15, 083031 (2013).

[81] I. Pižorn, V. Eisler, S. Andergassen, and M. Troyer, New J. Phys. 16, 073007 (2014)

[82] B. Lake, D. A. Tennant, J.-S. Caux, T. Barthel, U. Schollwöck, S. E. Nagler, and C. D. Frost, Phys. Rev. Lett. 111, 137205 (2013).

[83] A. C. Tiegel, S. R. Manmana, T. Pruschke, and A. Honecker, Phys. Rev. B 90, 060406(R) (2014).

[84] C. Lanczos, J. Res. Nat. Bur. Stand. 45, 255 (1950).

[85] E. Dagotto, Rev. Mod. Phys. 66, 763 (1994).

[86] R. Haydock, V. Heine, and M. J. Kelly, J. Phys. C 5, 2845 (1972).

[87] E. R. Gagliano and C. A. Balseiro, Phys. Rev. Lett. 59, 2999 (1987).

[88] A. Schmitt, K.-H. Mütter, M. Karbach, Y. Yu, and G. Müller, Phys. Rev. B 58, 5498 (1998).

[89] T. Sakai and M. Takahashi, Phys. Rev. B 42, 1090(R) (1990).

[90] S. R. White and I. Affleck, Phys. Rev. B 77, 134437 (2008).

[91] S. Trebst, H. Monien, C. J. Hamer, Z. Weihong, and R. R. P. Singh, Phys. Rev. Lett. 85, 4373 (2000).

[92] C. Knetter, K. P. Schmidt, M. Grüninger, and G. S. Uhrig, Phys. Rev. Lett. 87, 167204 (2001).

[93] M. Grüninger, M. Windt, T. Nunner, C. Knetter, K. P. Schmidt, G. S. Uhrig, T. Kopp, A. Freimuth, U. Ammerahl, B. Büchner, and A. Revcolevschi, J. Phys. Chem. Solids 63, 2167 (2002).

[94] K. P. Schmidt and G. S. Uhrig, Mod. Phys. Lett. B 19, 1179 (2005).

[95] P. Lemmens, M. Grove, M. Fischer, G. Güntherodt, V. N. Kotov, H. Kageyama, K. Onizuka, and Y. Ueda, Phys. Rev. Lett. 85, 2605 (2000).

[96] P. Corboz and F. Mila, Phys. Rev. B 87, 115144 (2013).

[97] S. Miyahara and K. Ueda, J. Phys. Soc. Jpn. 69 (Suppl B), 72 (2000).

[98] M. E. Zayed, C. Rüegg, J. Larrea, A. M. Läuchli, C. Panagopoulos, S. S. Saxena, M. Ellerby, D. F. McMorrow, T. Strässle, S. Klotz, G. Hamel, R. A. Sadykov, V. 
Pomjakushin, M. Boehm, M. Jiménez-Ruiz, A. Schneidewind, E. Pomjakushina, M. Stingaciu, K. Conder, and H. M. Rønnow, arXiv: 1603.02039 .

[99] D. C. Dender, P. R. Hammar, D. H. Reich, C. Broholm, and G. Aeppli, Phys. Rev. Lett. 79, 1750 (1997).

[100] M. Oshikawa and I. Affleck, Phys. Rev. Lett. 79, 2883 (1997).

[101] I. Affleck and M. Oshikawa, Phys. Rev. B 60, 1038 (1999).

[102] T. Asano, H. Nojiri, Y. Inagaki, J. P. Boucher, T. Sakon, Y. Ajiro, and M. Motokawa, Phys. Rev. Lett. 84, 5880 (2000).
[103] T. Asano, H. Nojiri, Y. Inagaki, T. Sakon, J.-P. Boucher, Y. Ajiro, and M. Motokawa, Phys. B (Amsterdam, Neth.) 329-333, 1213 (2003).

[104] T. Iitaka and T. Ebisuzaki, Phys. Rev. Lett. 90, 047203 (2003).

[105] I. Umegaki, H. Tanaka, T. Ono, H. Uekusa, and H. Nojiri, Phys. Rev. B 79, 184401 (2009).

[106] S. A. Zvyagin, Low Temp. Phys. 38, 819 (2012).

[107] M. Ozerov, M. Maksymenko, J. Wosnitza, A. Honecker, C. P. Landee, M. M. Turnbull, S. C. Furuya, T. Giamarchi, and S. A. Zvyagin, Phys. Rev. B 92, 241113(R) (2015). 